\title{
Environmental factors affecting pondweeds in water bodies of northwest Poland
}

\author{
Marek Merdalski*, Krzysztof Banaś \& Rafał Ronowski
}

Department of Plant Ecology, Faculty of Biology, University of Gdańsk, Wita Stwosza 59, 80-308 Gdańsk, Poland; ORCID: MM http:// orcid.org/0000-0003-2117-4889, KB http://orcid.org/0000-0002-2131-8400, RR http://orcid.org/0000-0002-5638-7131

*corresponding author (e-mail: marek.merdalski@ug.edu.pl)

\begin{abstract}
In 32 lakes, 19 watercourses and 11 estuaries located along the southern Baltic coast (NW Poland) taxa of Potomogeton and Stuckenia genera were determined on the basis of $981 / 0.1 \mathrm{~m}^{2}$ plant samples. Environmental factors affecting them were identified on the basis of 212 water samples and 272 sediment samples. Twenty-one Potamogeton taxa were found, including four hybrids and two Stuckenia species. Twenty-one pondweed species occurred in lakes, thirteen in watercourses and ten in estuaries. There were significant differences in environmental factors in particular types of water bodies $(p<0.001)$ except for the content of organic and mineral matter and of humic acids in the sediment.

There was a statistically significant difference $(\mathrm{p}<0.001)$ between the environmental factors affecting Potamogeton and Stuckenia, respectively, within each of the waterbody types studied. In lakes, Potamogeton occupied poorer habitats than Stuckenia, with lower conductivity, redox, PAR intensity, concentration of bicarbonates, calcium and chlorides and lower calcium content in the sediment. In watercourses, Potamogeton occurred in less coloured and less oxygenated waters than Stuckenia, but richer in $\mathrm{CO}_{2}$ and chlorides, better insolated and flowing faster. It also occupied less alkaline sediment, but of higher conductivity. In estuaries, Potamogeton occurred in waters with a relatively low concentration of chlorides and calcium than Stuckenia, lower $\mathrm{pH}$ and conductivity, but more coloured and, consequently, with lower PAR. C\&RT analysis showed mineral concentration (Ca ${ }^{2+}$, $\mathrm{Cl}^{-}, \mathrm{HCO}_{3}^{-}$) in the water, its conductivity, colour and flow to be the highest-ranking environmental factors affecting pondweeds.
\end{abstract}

Key words: Potamogeton, Stuckenia, pondweed, lakes, estuaries, watercourses, environmental factors

\section{Introduction}

The history of research concerning Potamogeton goes back to the turn of the $18^{\text {th }}$ and $19^{\text {th }}$ centuries. On the Polish territory, the research was conducted by Jan Fryderyk Wolfgang (1775-1859) and Stanisław Batyst Gorski (1802-1864). The first extensive monograph of the Potamogeton species was written by Hagström (1916) and was largely topical ever since (ZalewskaGałosz 2008). In the $20^{\text {th }}$ century, numerous scientists all over the world dealt with this group of plants, among them Fernald (1932), Ogden (1943), Haynes (1974), Reznicek \& Bobbette (1976), Haynes \& Hellquist (2000) in North America; Tur (1982) in South America; Miki (1937), Kadono (1982), Kaplan (2008), Wang et al. (2007) in Asia; Dandy (1937), Obermeyer (1966), Symoens et al. (1979), Kaplan \& Symoens (2005) in
Africa; Aston (1973) in Australia; Yuzepchuk (1934), Mäemets (1979, 1984), Tzvelev (1987), Kashina (1988), Volobaev (1993), Bobrov \& Chemeris (2009) in Russia and Wiegleb (1983, 1984), Brux et al. (1988), Preston (1995), Kaplan (2005, 2010), Zalewska-Gałosz (2002, 2008) in Europe. Their research brought numerous studies of the kind; however, they were usually fragmentary. It was only Wiegleb and Kaplan (1998) who wrote a monograph covering 69 species and 50 hybrids existing today. Their occurrence all over continents shows their high adaptability to changing environmental conditions. Recently, however, most academic papers dealt with taxonomy and its verification, together with the distribution of species (Preston 1993; Preston \& Stewart 1994; Afranowicz 2007; Chmara \& Bociag 2007; Kaplan 2008). As a result, subgenus Coleogeton was excluded from the Potamogeton genus and raised 
again to the rank of genus (Börner 1912; Holub 1984; Les \& Haynes 1996; Haynes et al. 1998; Zang et al. 2008). However, knowledge of environmental conditions in which Potamogeton species occur is still insufficient.

This paper aims at the identification of environmental conditions of Potamogeton and Stuckenia taxa, which in Poland occur along southern Baltic coast (NW Poland). Conditions of Potamogeton and Stuckenia occurrence in lakes, watercourses and estuaries were compared, and main environmental factors influencing their appearance in this type of water bodies were identified. The paper discusses empirical and conceptual models of correlation of Potamogeton and Stuckenia environmental factors.

\section{Material and methods}

Field research was conducted between 2008-2017 in 32 lakes, 19 watercourses and 11 estuaries (NW Poland, Fig. 1). Each site underwent a single observation in July, in the afternoon, with the same methodology. Samples were collected at random by a diver in a transect of approximately 250 metres, at one-metre-interval depth zones down to the lowest zone of plant occurrence (Chmara et al. 2015). In the area of $0.1 \mathrm{~m}^{2}$, coverage of individual taxa was identified, then all plants were collected and packed into sacks made of fine mesh (excluding samples of $P$. polygonifolius - noninvasive research at the Białogóra Nature Reserve). Altogether, 1474 samples with plants were collected. Plants were then segregated by taxa, dried and weighed. Samples which contained at least one taxon of Potamogeton or Stuckenia were used for further analysis (981 samples).
84 taxa altogether were identified in the samples, including 21 Potamogeton (17 species and 4 hybrids) and 2 Stuckenia. Key parts of specimens, like leaves, stems, stipules and inflorescence, were used to identify Potamogeton as described in the publications of Hagström (1916), Mądalski (1977), Wiegleb (1990), Zalewska-Gałosz (2008). Names of taxa were used in accordance with The Plant List (2013).

At the same depth zone of the transect where plant samples were collected, the diver collected sediment and water samples in order to describe the habitat of Potamogeton (212 water samples and 272 sediment samples). Water samples were put into polypropylene bottles $\left(0.5 \mathrm{dm}^{3}\right)$, while the sediment was collected in the form of a short core $\left(1 \mathrm{dm}^{3}\right)$ and then put into plastic ziplock bags. Measurements were taken in the water, for depth profiles of one metre. Temperature and oxygenation were measured with a WTW OXI 197i oxygen metre with the EOT 196 electrode. PAR at the sites where samples were collected was measured with LI-COR light metre with a flat sensor for incident radiation measurement LI-250 Light Meter, and the results were recalculated as a percentage of the light reaching water surface. Water flow was measured with Valeport flow meter, model 801 (flat) EM Flow Meter (the mean value three measurements for each depth zone where the plants occurred).

The research took into consideration aquatic environment factors generally recognised as important for the formation of plant communities, such as: depth, PAR intensity (van den Berg et al. 2003), oxygenation and temperature (Rooney \& Kalff 2000), reaction (Srivasta et al. 1995), conductivity (Toivonen \& Huttunen 1995), redox potential (in water and sediments), nutrient availa-

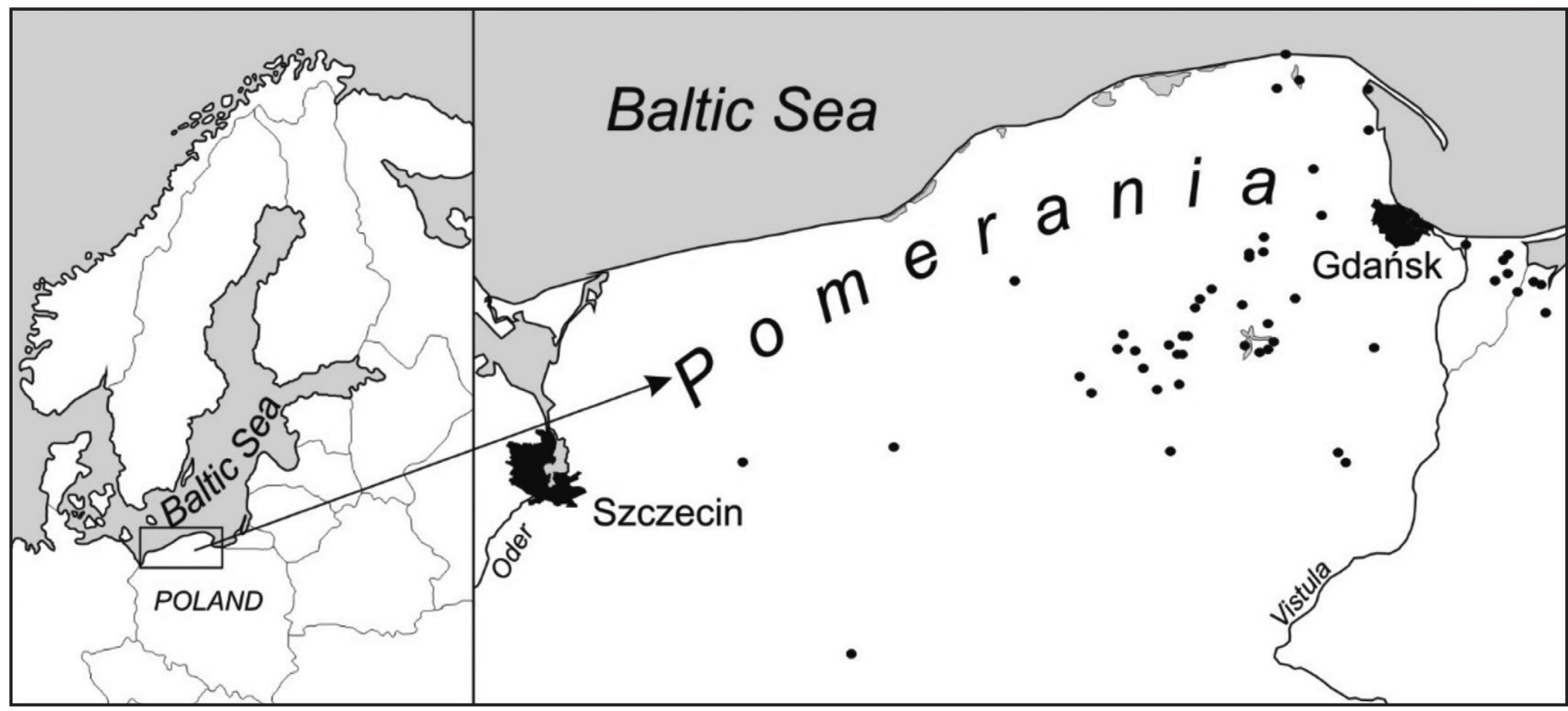

Fig. 1. Location of water bodies with pondweeds in north-western Poland 
bility (C, N, P; Murphy et al. 2003), water colour, concentration of humic acids, calcium and chlorides, and water flow (Chambers et al. 1991; Madsen et al. 2001). In the sediment, the following factors were checked: hydration, organic and mineral matter contents, reaction, conductivity, contents of humic acid and calcium, noncarbonate mineral matter, and the proportions of various granulation fractions (Lehmann et al. 1997; Gafny \& Gasith 1999).

In this paper, physical and chemical factors of the aquatic environment were assayed in the laboratory, following the works of Hermanowicz et al. (1999), and Eaton et al. (2005). Water and sediment conductivity was measured with LF 96 conductivity meter with a TETRACON 96 electrode, and the redox potential and $\mathrm{pH}-$ with a WTW 320/SET1 pH meter with respective glass METTLER electrode and SENTIX 97T electrode. Water colour was identified by comparative method according to platinum-cobalt scale. The concentration of dissolved forms of inorganic carbon (DIC; $\mathrm{CO}_{2}, \mathrm{HCO}_{3}{ }^{-}$and $\mathrm{CO}_{3}{ }^{2-}$ ) in water was assessed by titration, concentration of calcium in water-by complexometric EDTA method, and in sediments after $\mathrm{Ca}^{2+}$ extraction - with hydrochloric acid (1:1). Humic acids concentration was measured with UV-VIS spectrophotometer at the wave length of $330 \mathrm{~nm}$ according to Moore $(1985,1987)$ and Górniak (1996), while in the sediment after earlier extraction - in $0.5 \mathrm{n} \mathrm{NaOH}$. Total nitrogen, total phosphorus and chlorides were estimated with a MERCK Spectroquant cuvette test on the UV-VIS spectrophotometer. Samples for phosphorus assays were earlier mineralised in the environment of sulphuric and nitric acids in Microwave Digestion System - Start D (Milestone). Hydration was measured on the basis of the difference between fresh and dry sediment, after drying it at $105^{\circ} \mathrm{C}$ to dry solids. Organic matter content was calculated from the difference between the sediment weight before and after combustion at $550^{\circ} \mathrm{C}$ in the Thermolyne 62700 muffle furnace. Non-carbonate matter content $\left(\mathrm{SiO}_{2}\right)$ in the sediment was estimated after previous dissolution of a weighed portion of the incinerated sediment in $1: 1 \mathrm{HCl}$ solution. The share of sediment fractions of different granulations: $<0.1 \mathrm{~mm}, 0.1-0.25 \mathrm{~mm}, 0.25-0.5 \mathrm{~mm}$, $0.5-1 \mathrm{~mm}, 1-2 \mathrm{~mm}$ and $>2 \mathrm{~mm}$ was calculated as weight percentage of dry sediment after sifting through sieves with specified mesh size.

The database was constructed as a matrix in which each of the species was entered into a separate column (84) and each line represented a single sample (981 samples of Potamogeton). Individual fields of the matrix were filled in a binary way (yes -1 , no -0 ). Next, the occurrence of individual types of Potamogeton was calculated.

Another matrix with the same structure was produced for 981 samples in terms of 14 water properties and 10 sediment properties, where - like in the previous database - the line represents the next sample and the column - the identified environmental feature.

The STATISTICA 12.0 software was used for data analysis to calculate arithmetic average, standard deviation, minimum and maximum value for each of the studied features, for each species, and type of water body. In order to establish the length of the distribution gradient, CANOCO 4.5 software was used for detrended correspondence analysis (DCA, gradient length $=1.304)$. In order to identify main factors affecting species and genus distribution (Potamogeto and Stuckenia) in the water bodies studied, the principal component analysis (PCA) was conducted on the basis of calculations made by CANOCO 4.5 (Hastie et al. 2001; Ter Braak \& Šmilauer 2002). Before analyses, the scaling was focused on inter-species correlations, samples were cantered and standardised, but the data were not transformed. The selection of environmental variables was automatic.

The analysis of relationships between the occurrence of Potamogeton and Stuckenia and environmental factors in the water bodies was performed by means of the Classification and Regression Tree (C\&RT) with Gini impurity $\left(\mathrm{I}_{\mathrm{G}}(\mathrm{f})\right.$ in the Statistica 12.0 software, in accordance with the recommendations given by Bell (1999); Austin (2007) and Olden et al. (2008). Predictive validity of environmental factors was determined according to the value of the validity coefficient.

\section{Results}

\subsection{Pondweeds in the study area}

21 pondweed taxa were found in the lakes, 13 in the watercourses and 10 in the estuaries (Table 1). Stuckenia pectinata, Potamogeton perfoliatus, P. obtusifolius, $P$. crispus, $P$. natans, $P$. lucens and $P$. compressus occurred in each of the waterbody categories. Stuckenia filiformis, $P$. gramineus, $P$. polygonifolius, $P$. $\times$ nitens, $P$. $\times$ salicifolius and $P$. $\times$ angustifolius, inhabited only lakes, while the occurrence of $P$. nodosus and $P$. $\times$ sparganifolius was confined to the watercourses.

Most pondweeds occurred at the depths of up to two metres. The water in such places was slightly alkaline (pH 7.5-8.5), rich in nitrogen, phosphorus, carbon (DIC) and calcium, and slightly coloured (Table 2). Chloride concentration varied significantly, from $0.5 \mathrm{mg}$ $\mathrm{Cl}^{-} \mathrm{dm}^{-3}$ in the lakes, $116.3 \mathrm{mg} \mathrm{Cl}^{-} \mathrm{dm}^{-3}$ in the estuary section of the River Vistula up to $5121,4 \mathrm{mg} \mathrm{Cl}^{-} \mathrm{dm}^{-3}$ in the Bay of Puck. The water was well oxygenated, but the photosynthetic irradiation was low. The sediment was $\mathrm{pH}$-neutral, poorly hydrated, with a low redox. It contained little organic matter but was rich in $\mathrm{SiO}_{2}$ and calcium (Table 3). 
Table 1. Number of pondweed samples in the studied waterbodies by category

\begin{tabular}{|c|c|c|c|c|}
\hline \multirow[b]{2}{*}{ Species } & \multicolumn{4}{|c|}{ Number of samples } \\
\hline & lakes & watercourses & estuaries & total \\
\hline S. pectinata (L.) Börner & 161 & 51 & 54 & 266 \\
\hline P. perfoliatus L. & 97 & 47 & 34 & 178 \\
\hline P. obtusifolius Mert. \& W. D. J. Koch & 70 & 46 & 18 & 134 \\
\hline P. friesii Rupr. & 114 & 6 & - & 118 \\
\hline P. crispus $\mathrm{L}$. & 48 & 28 & 32 & 108 \\
\hline P. natans $\mathrm{L}$. & 79 & 8 & 16 & 103 \\
\hline P. lucens $\mathrm{L}$. & 29 & 26 & 18 & 73 \\
\hline P. gramineus L. & 62 & - & - & 62 \\
\hline P. compressus L. & 31 & 16 & 3 & 50 \\
\hline P. trichoides Cham. \& Schltdl. & 17 & - & 23 & 40 \\
\hline P. praelongus Wulfen & 29 & - & 4 & 33 \\
\hline P. alpinus Balb. & 8 & 23 & - & 31 \\
\hline P. berchtoldii Fieber & 16 & 2 & - & 18 \\
\hline P. pusillus L. & 13 & - & 2 & 15 \\
\hline P. $\times$ nitens Weber & 14 & - & - & 14 \\
\hline P. $\times$ salicifolius Wolfg. & 13 & - & - & 13 \\
\hline P. rutilus Wolfg. & 7 & 4 & - & 11 \\
\hline S. filiformis (Pers.) Börner & 10 & - & - & 10 \\
\hline P. polygonifolius Pourr. & 6 & - & - & 6 \\
\hline P. nodosus Poir. & - & 5 & - & 5 \\
\hline P. $\times$ angustifolius J. Presl & 5 & - & - & 5 \\
\hline P. $\times$ sparganifolius Laest. ex Fr. & - & 3 & - & 3 \\
\hline P. acutifolius Link ex Roem. \& Schult. & 1 & - & - & 1 \\
\hline
\end{tabular}

Table 2. Water traits in the studied pondweed habitats

\begin{tabular}{|c|c|c|c|c|c|c|c|c|c|}
\hline Species & $\mathrm{pH}$ & $\begin{array}{l}\text { Conductivity } \\
{\left[\mu \mathrm{S} \mathrm{cm}^{-1}\right]}\end{array}$ & $\begin{array}{c}\text { Water } \\
\text { colour } \\
{\left[\mathrm{mg} \mathrm{Pt}^{-3}\right.} \\
\left.\mathrm{dm}^{-3}\right]\end{array}$ & $\begin{array}{c}\mathrm{Ca}^{2+} \\
{\left[\mathrm{mg} \mathrm{dm}^{-3}\right]}\end{array}$ & $\begin{array}{l}\mathrm{N}_{\text {tot. }} \\
{[\mathrm{mg}} \\
\left.\mathrm{dm}^{-3}\right]\end{array}$ & $\begin{array}{c}\mathrm{P}_{\text {tot. }} \\
{[\mathrm{mg}} \\
\left.\mathrm{dm}^{-3}\right]\end{array}$ & $\begin{array}{c}\mathrm{Cl}^{-} \\
{\left[\mathrm{mg} \mathrm{dm}^{-3}\right]}\end{array}$ & $\begin{array}{l}\text { PAR } \\
{[\%]}\end{array}$ & $\begin{array}{c}\text { Oxygenation } \\
{[\%]}\end{array}$ \\
\hline S. pectinata & $8.02 \pm 0.44$ & $384 \pm 309$ & $22 \pm 18$ & $55.3 \pm 28.0$ & $1.5 \pm 1.1$ & $0.3 \pm 0.2$ & $259.2 \pm 910.1$ & $30.0 \pm 20.2$ & $107.2 \pm 107.2$ \\
\hline P. perfoliatus & $7.96 \pm 0.40$ & $371 \pm 273$ & $28 \pm 22$ & $53.4 \pm 23.4$ & $1.5 \pm 1.2$ & $0.4 \pm 0.2$ & $21.8 \pm 33.0$ & $30.7 \pm 21.5$ & $110.7 \pm 19.6$ \\
\hline P. obtusifolius & $8.10 \pm 0.33$ & $331 \pm 233$ & $25 \pm 22$ & $51.1 \pm 17.4$ & $2.4 \pm 1.7$ & $0.4 \pm 0.2$ & $16.3 \pm 21.5$ & $29.1 \pm 22.0$ & $102.5 \pm 32.4$ \\
\hline P. friesii & $7.96 \pm 0.29$ & $223 \pm 35.9$ & $12 \pm 6$ & $42.9 \pm 8.3$ & $1.3 \pm 0.7$ & $0.2 \pm 0.1$ & $4.6 \pm 2.5$ & $27.4 \pm 22.8$ & $110.8 \pm 22.6$ \\
\hline P. crispus & $8.01 \pm 0.38$ & $394 \pm 305$ & $29 \pm 33$ & $56.7 \pm 29.3$ & $1.7 \pm 1.3$ & $0.4 \pm 0.2$ & $52.5 \pm 265.3$ & $28.4 \pm 24.5$ & $96.9 \pm 38.7$ \\
\hline P. natans & $7.75 \pm 0.70$ & $258 \pm 240$ & $27 \pm 28$ & $36.6 \pm 28.2$ & $1.5 \pm 0.8$ & $0.3 \pm 0.2$ & $16.3 \pm 27.3$ & $34.0 \pm 22.2$ & $96.0 \pm 28.9$ \\
\hline P. lucens & $7.81 \pm 0.32$ & $466 \pm 332$ & $31 \pm 26$ & $59.4 \pm 24.0$ & $1.5 \pm 0.8$ & $0.4 \pm 0.2$ & $25.2 \pm 28.1$ & $17.5 \pm 15.6$ & $83.7 \pm 34.6$ \\
\hline P. gramineus & $7.85 \pm 0.24$ & $160 \pm 44.1$ & $10 \pm 6$ & $28.1 \pm 7.4$ & $1.2 \pm 0.6$ & $0.2 \pm 0.1$ & $4.4 \pm 1.8$ & $49.8 \pm 25.1$ & $114.8 \pm 9.7$ \\
\hline P. compressus & $8.09 \pm 0.35$ & $345 \pm 303$ & $31 \pm 10$ & $46.5 \pm 16.3$ & $2.6 \pm 1.2$ & $0.4 \pm 0.1$ & $17.4 \pm 20.9$ & $15.2 \pm 12.3$ & $96.1 \pm 22.8$ \\
\hline P. trichoides & $7.87 \pm 0.41$ & $559 \pm 319$ & $34 \pm 16$ & $76.6 \pm 37.4$ & $2.3 \pm 0.7$ & $0.4 \pm 0.1$ & $37.9 \pm 26.7$ & $14.5 \pm 12.8$ & $83.3 \pm 27.9$ \\
\hline P. praelongus & $8.01 \pm 0.23$ & $209 \pm 288$ & $22 \pm 10$ & $29.1 \pm 21.4$ & $2.1 \pm 1.5$ & $0.7 \pm 0.5$ & $10.7 \pm 22.4$ & $18.7 \pm 7.7$ & $102.8 \pm 23.0$ \\
\hline P. alpinus & $7.80 \pm 0.33$ & $244 \pm 40.3$ & $15 \pm 5$ & $46.1 \pm 9.3$ & $1.8 \pm 1.5$ & $0.3 \pm 0.2$ & $4.0 \pm 1.9$ & $40.6 \pm 22.7$ & $105.5 \pm 21.7$ \\
\hline P. berchtoldii & $8.16 \pm 0.35$ & $249 \pm 76.2$ & $12 \pm 6$ & $42.1 \pm 10.8$ & $1.0 \pm 0.8$ & $0.2 \pm 0.1$ & $10.2 \pm 8.4$ & $23.7 \pm 17.0$ & $113.3 \pm 22.0$ \\
\hline P. pusillus & $8.06 \pm 0.56$ & $237 \pm 186$ & $17 \pm 10$ & $43.7 \pm 35.4$ & $2.3 \pm 1.5$ & $0.3 \pm 0.2$ & $9.8 \pm 15.6$ & $26.2 \pm 9.5$ & $108.1 \pm 14.6$ \\
\hline P. $\times$ nitens & $7.9 \pm 0.19$ & $189 \pm 13.7$ & $9 \pm 5$ & $31.9 \pm 2.8$ & $0.8 \pm 0.7$ & $0.2 \pm 0.1$ & $5.2 \pm 1.9$ & $28.0 \pm 11.2$ & $122.6 \pm 6.4$ \\
\hline P. $\times$ salicifolius & $8.09 \pm 0.34$ & $196 \pm 22.7$ & $6 \pm 4$ & $35.0 \pm 3.1$ & $1.1 \pm 0.5$ & $0.1 \pm 0.1$ & $4.8 \pm 6.2$ & $17.4 \pm 1.6$ & $105.9 \pm 0.3$ \\
\hline P. rutilus & $7.71 \pm 0.13$ & $165 \pm 93.0$ & $11 \pm 3$ & $32.3 \pm 22.6$ & $2.3 \pm 2.0$ & $0.3 \pm 0.2$ & $3.3 \pm 0.3$ & $30.9 \pm 22.8$ & $110.8 \pm 30.2$ \\
\hline S. filiformis & $7.98 \pm 0.19$ & $206 \pm 0.7$ & $10 \pm 3$ & $39.5 \pm 8.6$ & $0.9 \pm 0.1$ & $0.1 \pm 0.1$ & $5.3 \pm 0.3$ & $39.5 \pm 6.2$ & $121.4 \pm 3.9$ \\
\hline P. poligonifolius & $4.89 \pm 0.1$ & $48.8 \pm 5.4$ & $275 \pm 137$ & $1.2 \pm 0.1$ & $0.8 \pm 0.1$ & $0.2 \pm 0.1$ & $8.7 \pm 2.2$ & $12.8 \pm 8.3$ & $48.9 \pm 3.7$ \\
\hline P. nodosus & $7.67 \pm 0.03$ & $367 \pm 0.0$ & $25 \pm 2$ & $62.2 \pm 0.3$ & $0.9 \pm 0.1$ & $0.2 \pm 0.1$ & $9.2 \pm 0.4$ & $45.4 \pm 3.0$ & $65.1 \pm 0.0$ \\
\hline P. $\times$ angustifolius & $7.78 \pm 0.0$ & $155 \pm 0.0$ & $12 \pm 0$ & $27.7 \pm 0.0$ & $1.7 \pm 0.0$ & $0.1 \pm 0.0$ & $1.6 \pm 0.0$ & $19.2 \pm 0.0$ & $111.7 \pm 0.0$ \\
\hline P. $\times$ sparganifolius & $8.27 \pm 0.0$ & $189 \pm 0.0$ & $15 \pm 0$ & $44.9 \pm 0.0$ & $0.9 \pm 0.0$ & $0.4 \pm 0.0$ & $6.7 \pm 0.0$ & $43.3 \pm 0.0$ & $147.5 \pm 0.0$ \\
\hline P. acutifolius & 8.16 & 240.0 & 35 & 41.04 & 3.6 & 0.34 & 9.44 & 6.57 & 106.1 \\
\hline
\end{tabular}

Explanations: see Fig. 2 
Table 3. Sediment traits in the studied pondweed habitats

\begin{tabular}{lcccccc}
\hline Species & Depth [m] & $\begin{array}{c}\text { Organic } \\
\text { matter } \\
{[\%]}\end{array}$ & $\begin{array}{c}\text { Hydration } \\
{[\%]}\end{array}$ & $\mathrm{pH}$ & $\begin{array}{c}\text { Redox } \\
\text { potential } \\
{[\mathrm{mV}]}\end{array}$ & $\begin{array}{c}\mathrm{Ca}^{2+} \\
{\left[\mathrm{mg} \mathrm{g}^{-1} \text { d.w.] }\right.}\end{array}$ \\
\hline S. pectinata & $1.3 \pm 0.9$ & $6.2 \pm 8.5$ & $34.9 \pm 25.1$ & $7.19 \pm 0.3$ & $-242 \pm 104$ & $57.8 \pm 96.5$ \\
P. perfoliatus & $1.1 \pm 0.9$ & $4.5 \pm 6.7$ & $30.6 \pm 22.6$ & $7.18 \pm 0.37$ & $-248 \pm 101$ & $46.9 \pm 85.0$ \\
P. obtusifolius & $1.3 \pm 1.1$ & $9.6 \pm 11.9$ & $37.8 \pm 27.4$ & $7.22 \pm 0.32$ & $-245 \pm 121$ & $55.6 \pm 87.0$ \\
P. friesii & $1.9 \pm 1.2$ & $10.9 \pm 9.8$ & $37.0 \pm 30.0$ & $7.18 \pm 0.23$ & $-244 \pm 70$ & $152.8 \pm 131.0$ \\
P. crispus & $1.7 \pm 1.6$ & $12.1 \pm 14.9$ & $39.1 \pm 27.0$ & $7.15 \pm 0.34$ & $-243 \pm 116$ & $45.0 \pm 80.0$ \\
P. natans & $1.0 \pm 0.6$ & $15.5 \pm 25.4$ & $42.2 \pm 31.7$ & $6.94 \pm 0.39$ & $-156 \pm 143$ & $36.3 \pm 77.1$ \\
P. lucens & $1.6 \pm 0.7$ & $10.8 \pm 12.1$ & $53.9 \pm 27.5$ & $7.13 \pm 0.38$ & $-258 \pm 111$ & $31.8 \pm 42.1$ \\
P. gramineus & $0.8 \pm 1.1$ & $1.4 \pm 2.2$ & $18.3 \pm 12.8$ & $6.99 \pm 0.29$ & $-131 \pm 185$ & $2.7 \pm 2.7$ \\
P. compressus & $1.3 \pm 0.5$ & $4.5 \pm 5.4$ & $42.1 \pm 19.7$ & $7.21 \pm 0.32$ & $-288 \pm 76$ & $88.3 \pm 94.7$ \\
P. trichoides & $1.1 \pm 0.5$ & $10.4 \pm 13.2$ & $48.3 \pm 25.5$ & $6.96 \pm 0.32$ & $-322 \pm 56$ & $40.8 \pm 76.4$ \\
P. praelongus & $2.1 \pm 0.7$ & $33.2 \pm 22.0$ & $76.2 \pm 34.0$ & $6.91 \pm 0.21$ & $-225 \pm 104$ & $14.0 \pm 21.8$ \\
P. alpinus & $1.0 \pm 1.0$ & $6.0 \pm 11.9$ & $25.8 \pm 27.0$ & $7.22 \pm 0.21$ & $-147 \pm 130$ & $8.8 \pm 6.7$ \\
P. berchtoldii & $2.3 \pm 1.5$ & $3.0 \pm 5.6$ & $17.0 \pm 27.1$ & $7.21 \pm 0.16$ & $-251 \pm 91$ & $31.5 \pm 73.4$ \\
P. pusillus & $1.4 \pm 1.0$ & $5.5 \pm 6.4$ & $45.3 \pm 31.0$ & $6.89 \pm 0.39$ & $-192 \pm 133$ & $22.8 \pm 46.9$ \\
P. $\times$ nitens & $2.1 \pm 1.0$ & $1.4 \pm 1.0$ & $23.7 \pm 19.3$ & $7.14 \pm 0.35$ & $-211 \pm 93$ & $5.5 \pm 5.6$ \\
P. $\times$ salicifolius & $4.2 \pm 0.3$ & $2.2 \pm 3.4$ & $36.4 \pm 11.8$ & $7.25 \pm 0.18$ & $-199 \pm 38$ & $3.0 \pm 1.2$ \\
P. rutilus & $2.2 \pm 1.4$ & $2.9 \pm 1.0$ & $15.6 \pm 11.5$ & $6.99 \pm 0.34$ & $-121 \pm 175$ & $1.7 \pm 0.8$ \\
S. filiformis & $0.5 \pm 0.4$ & $3.9 \pm 10.9$ & $15.8 \pm 27.1$ & $7.21 \pm 0.31$ & $-41 \pm 96$ & $13.2 \pm 36.6$ \\
P. poligonifolius & $0.3 \pm 0.1$ & $29.4 \pm 25.6$ & $76.8 \pm 18.1$ & $5.34 \pm 0.29$ & $-187 \pm 79$ & $4.9 \pm 4.5$ \\
P. nodosus & $0.5 \pm 0.1$ & $2.6 \pm 3.6$ & $30.1 \pm 14.2$ & $7.23 \pm 0.29$ & $-573 \pm 173$ & $6.0 \pm 0.1$ \\
P. $\times$ angustifolius & $1.9 \pm 0.3$ & $2.0 \pm 0$ & $26.8 \pm 0$ & $6.8 \pm 0$ & $-144 \pm 0$ & $0.9 \pm 0$ \\
P. $\times$ sparganifolius & $1.1 \pm 0.2$ & $38.8 \pm 0$ & $16.1 \pm 0$ & $7.45 \pm 0$ & $-259 \pm 0$ & $6.5 \pm 0$ \\
P. acutifolius & 1.6 & 4.28 & 58.04 & 7.24 & -308 & 232.15 \\
\hline & & & & & & \\
\hline
\end{tabular}

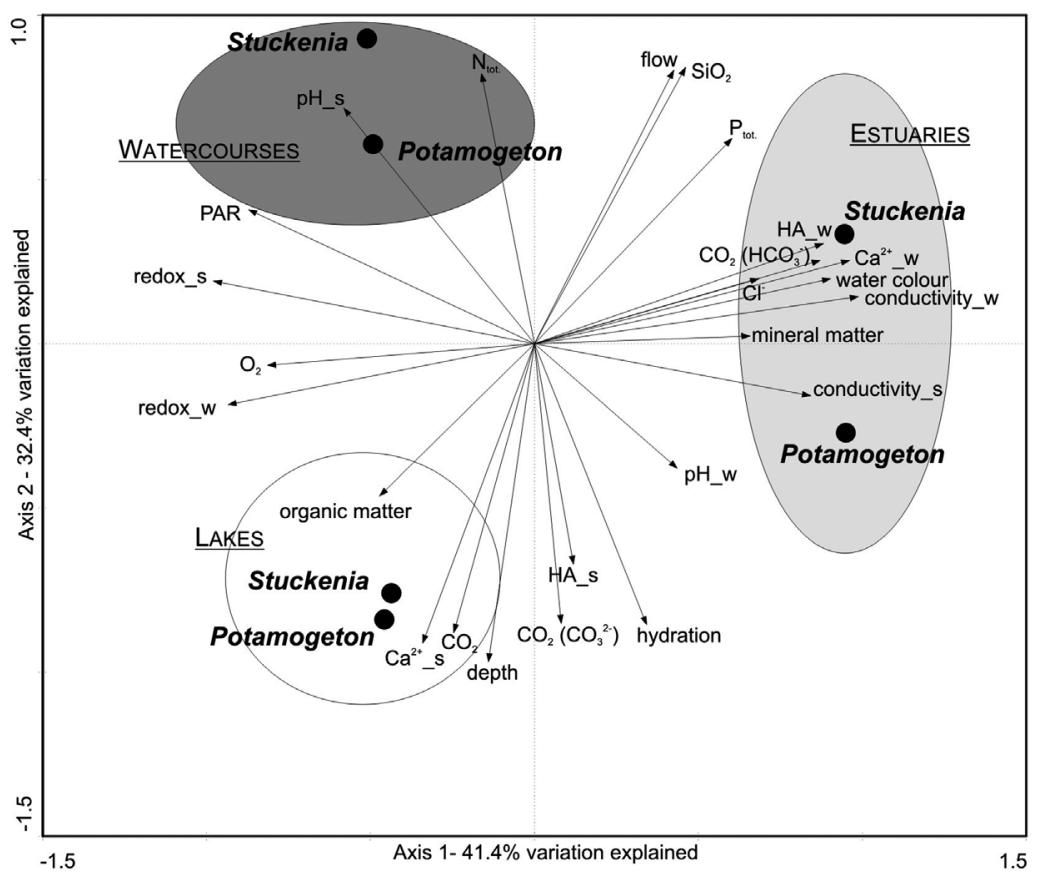

Fig. 2. Model of the relationship between pondweed distribution and PCA-ranked environmental factors in the studied lakes, watercourses and estuaries

Explanations: $\mathrm{HA}$ - humic acids, $\mathrm{N}_{\text {tot. }}$ - total nitrogen, $\mathrm{P}_{\text {tot. }}$ - total phosphorus, PAR - photosynthetically active radiation

\begin{tabular}{lccccc}
\hline \multicolumn{7}{c}{ Summary of PCA analysis } & & & \\
\hline Axes & 1 & 2 & 3 & 4 & Total inertia \\
Eigenvalues: & 0.414 & 0.324 & 0.175 & 0.071 & 1.000 \\
Cumulative percentage variance of species data: & 41.4 & 73.8 & 91.3 & 98.4 & 1.000 \\
Sum of all eigenvalues & & & & & \\
\hline
\end{tabular}


Table 4. Environmental factors and differences in water and sediment traits for the studied waterbody types

\begin{tabular}{|c|c|c|c|c|c|c|c|c|c|}
\hline \multirow[b]{2}{*}{ Trait } & \multicolumn{2}{|c|}{$\begin{array}{l}\text { Lakes } \\
n=629\end{array}$} & \multicolumn{2}{|c|}{$\begin{array}{l}\text { Watercourses } \\
n=196\end{array}$} & \multicolumn{2}{|c|}{$\begin{array}{c}\text { Estuaries } \\
n=156\end{array}$} & \multicolumn{3}{|c|}{$\begin{array}{c}\text { Statistical } \\
\text { significance }\end{array}$} \\
\hline & mean $\pm \mathrm{SD}$ & $\min -\max$ & mean $\pm \mathrm{SD}$ & $\min -\max$ & mean $\pm \mathrm{SD}$ & $\min -\max$ & $\mathrm{a}$ & $\mathrm{b}$ & $\mathrm{c}$ \\
\hline Depth [m] & $1.8 \pm 1.3$ & $0.0-6.3$ & $0.7 \pm 0.6$ & $0.1-2.4$ & $1.1 \pm 0.6$ & $0.3-2.5$ & $* * *$ & $* * *$ & $* *$ \\
\hline \multicolumn{10}{|c|}{ water traits } \\
\hline $\mathrm{pH}$ & $7.9 \pm 0.5$ & $4.8-8.9$ & $7.8 \pm 0.4$ & $7.3-8.4$ & $8.0 \pm 0.5$ & $6.9-8.9$ & $* *$ & - & $* *$ \\
\hline Conductivity $\left[\mu \mathrm{S} \mathrm{cm}^{-1}\right]$ & $193.8 \pm 66.1$ & $34.5-336$ & $278.2 \pm 66.2$ & $189.1-575$ & $905.1 \pm 164.6$ & $451-1136$ & $* * *$ & $* * *$ & $* * *$ \\
\hline Redox $[\mathrm{mV}]$ & $60 \pm 82$ & $-120-395$ & $53 \pm 89$ & $-86-322$ & $17 \pm 132$ & $-276-255$ & - & $* * *$ & $* * *$ \\
\hline Colour [mg Pt dm $\left.{ }^{-3}\right]$ & $17 \pm 31$ & $2-400$ & $22 \pm 11$ & $8-45$ & $52 \pm 32$ & $15-160$ & - & $* * *$ & $* * *$ \\
\hline $\mathrm{HA}\left[\mathrm{mg} \mathrm{dm}^{-3}\right]$ & $2.7 \pm 4.1$ & $0.3-45.1$ & $3.8 \pm 1.7$ & $1.2-8.3$ & $6.3 \pm 3.6$ & $2.2-17.1$ & $* *$ & $* * *$ & $* * *$ \\
\hline $\mathrm{CO}_{2}\left[\mathrm{mg} \mathrm{dm}^{-3}\right]$ & $8.0 \pm 6.4$ & $0.1-41.4$ & $5.4 \pm 4.2$ & $1.3-24.2$ & $5.1 \pm 3.2$ & $0.7-11.4$ & $* * *$ & $* * *$ & - \\
\hline $\mathrm{CO}_{3}^{2-}\left[\mathrm{mg} \mathrm{CO}_{2} \mathrm{dm}^{-3}\right]$ & $9.5 \pm 10.3$ & $0.0-62.9$ & $4.9 \pm 5.2$ & $0.0-26.4$ & $8.7 \pm 6.4$ & $0.0-18.5$ & $* * *$ & - & $* * *$ \\
\hline $\mathrm{HCO}_{3}^{-}\left[\mathrm{mg} \mathrm{CO}_{2} \mathrm{dm}^{-3}\right]$ & $61.0 \pm 26.4$ & $7.0-140.8$ & $107.8 \pm 42.6$ & $59.0-286.0$ & $180.9 \pm 58.9$ & $92.4-311.1$ & $* * *$ & $* * *$ & $* * *$ \\
\hline $\mathrm{Ca}^{2+}\left[\mathrm{mg} \mathrm{dm}^{-3}\right]$ & $35.2 \pm 13.1$ & $1.0-69.2$ & $51.5 \pm 11.0$ & $41.0-94.5$ & $96.6 \pm 16.9$ & $78.8-131.5$ & $* * *$ & $* * *$ & $* * *$ \\
\hline $\mathrm{N}_{\text {tot. }}\left[\mathrm{mg} \mathrm{N} \mathrm{dm}^{-3}\right]$ & $1.3 \pm 0.9$ & $0.3-4.0$ & $1.9 \pm 1.6$ & $0.3-5.3$ & $1.5 \pm 0.9$ & $0.5-4.8$ & $* * *$ & - & $* *$ \\
\hline $\mathrm{P}_{\text {tot. }}\left[\mathrm{mg} \mathrm{P} \mathrm{dm}^{-3}\right]$ & $0.2 \pm 0.2$ & $0.0-1.3$ & $0.4 \pm 0.2$ & $0.1-0.8$ & $0.5 \pm 0.2$ & $0.1-1.5$ & $* * *$ & $* * *$ & $* *$ \\
\hline $\mathrm{Cl}^{-}\left[\mathrm{mg} \mathrm{dm}^{-3}\right]$ & $6.0 \pm 5.8$ & $0.5-50.3$ & $6.1 \pm 2.7$ & $2.0-16.3$ & $495.1 \pm 1156.2$ & $47.6-5121.4$ & - & $* * *$ & $* * *$ \\
\hline PAR [\%] & $28.7 \pm 20.1$ & $1.5-100.0$ & $42.0 \pm 22.2$ & $0.4-100.0$ & $18.0 \pm 21.8$ & 0.1-100.0 & $* * *$ & $* * *$ & $* * *$ \\
\hline $\mathrm{O}_{2}[\%]$ & $111.2 \pm 19.0$ & $12.3-161.2$ & $101.7 \pm 30.6$ & $35.9-147.5$ & $71.1 \pm 30.3$ & $7.6-117.9$ & $* * *$ & $* * *$ & $* * *$ \\
\hline Flow $\left[\mathrm{ms}^{-1}\right]$ & 0 & 0 & $0.2 \pm 0.1$ & $0-0.5$ & $0.1 \pm 0.2$ & $0-0.6$ & $* * *$ & $* * *$ & $* * *$ \\
\hline \multicolumn{10}{|c|}{ sediment traits } \\
\hline $\mathrm{pH}$ & $7.1 \pm 0.4$ & $5.1-8.2$ & $7.3 \pm 0.3$ & $6.7-8.0$ & $7.1 \pm 0.3$ & $6.5-7.5$ & $* * *$ & - & $* * *$ \\
\hline Conductivity $\left[\mu \mathrm{S} \mathrm{cm}^{-1}\right]$ & $281.2 \pm 229$ & $41.1-2150$ & $349 \pm 439$ & $42.3-1435$ & $636.3 \pm 355.4$ & $40.0-1428$ & $*$ & $* * *$ & $* * *$ \\
\hline Redox $[\mathrm{mV}]$ & $-210 \pm 118$ & $-352-256$ & $-195 \pm 1270$ & $-650-267$ & $-329 \pm 105$ & $-460-33$ & - & $* * *$ & $* * *$ \\
\hline $\mathrm{Ca}^{2+}\left[\mathrm{mg} \mathrm{g}^{-1}\right.$ d.w. $]$ & $73.0 \pm 109.8$ & $0.4-376.9$ & $17.9 \pm 35.6$ & $1.0-188.4$ & $23.7 \pm 31.1$ & $0.7-153.0$ & $* * *$ & $* * *$ & - \\
\hline $\mathrm{HA}\left[\mathrm{mg} \mathrm{g}^{-1}\right.$ d.w.] & $8.1 \pm 13.5$ & $0.1-135.1$ & $5.9 \pm 13.3$ & $0.2-60.4$ & $9.1 \pm 9.9$ & $0.0-33.1$ & - & - & - \\
\hline Hydration [\%] & $39.1 \pm 31.4$ & $0.4-99.9$ & $29.2 \pm 20.5$ & $0.8-83.2$ & $43.0 \pm 25.7$ & $13.1-92.7$ & $* *$ & - & $* * *$ \\
\hline Organic matter [\%] & $10.8 \pm 16.1$ & $0.3-92.8$ & $8.0 \pm 12.9$ & $0.3-38.9$ & $7.7 \pm 8.2$ & $0.2-28.2$ & - & - & - \\
\hline Mineral matter [\%] & $89.2 \pm 16.1$ & $7.2-99.7$ & $89.6 \pm 19.2$ & $38.1-99.7$ & $92.3 \pm 8.2$ & $71.9-99.8$ & - & - & - \\
\hline $\mathrm{SiO}_{2}[\%]$ & $66.1 \pm 35.8$ & $0.2-100.3$ & $78.7 \pm 24.1$ & $32.4-98.4$ & $78.9 \pm 18.4$ & $29.8-98.6$ & $* * *$ & $* *$ & - \\
\hline Fraction $>2 \mathrm{~mm}[\%]$ & $5.6 \pm 12.2$ & $0.0-76.8$ & $10.2 \pm 15.2$ & $0.0-62.4$ & $2.0 \pm 5.3$ & $0.0-19.6$ & $* * *$ & $*$ & $* * *$ \\
\hline $1-2 \mathrm{~mm}[\%]$ & $3.3 \pm 4.3$ & $0.0-20.2$ & $4.8 \pm 4.2$ & $0.0-14.8$ & $2.4 \pm 3.9$ & $0.0-13.5$ & $* *$ & - & $* * *$ \\
\hline $0.5-1 \mathrm{~mm}[\%]$ & $8.7 \pm 10.0$ & $0.0-37.3$ & $16.8 \pm 12.9$ & $0.0-57.4$ & $7.8 \pm 12.1$ & $0.0-43.6$ & $* * *$ & - & $* * *$ \\
\hline $0.25-0.5 \mathrm{~mm}[\%]$ & $22.4 \pm 17.6$ & $0.0-62.3$ & $31.1 \pm 17.2$ & $0.0-65.2$ & $16.3 \pm 20.4$ & $0.0-64.4$ & $* * *$ & $*$ & $* * *$ \\
\hline $0.1-0.25 \mathrm{~mm}[\%]$ & $29.4 \pm 19.4$ & $0.0-83.4$ & $26.7 \pm 19.7$ & $0.0-84.5$ & $24.5 \pm 19.5$ & $0.0-68.8$ & - & $*$ & $* * *$ \\
\hline$<0.1 \mathrm{~mm}[\%]$ & $30.3 \pm 34.5$ & $0.0-100.0$ & $10.0 \pm 20.0$ & $0.1-100.0$ & $46.2 \pm 37.4$ & $0.1-100.0$ & $* * *$ & $* * *$ & $* * *$ \\
\hline
\end{tabular}

Explanations: statistically significant differences between the studied lakes and watercourses (a), lakes and estuaries (b), watercourses and estuaries (c); - no difference, ${ }^{*} \mathrm{p} \leq 0.05,{ }^{* *} \mathrm{p} \leq 0.01,{ }^{* * *} \mathrm{p} \leq 0.001$ (post-hoc Tukey's test), $\mathrm{n}$ - number of samples, HA - humic acids, $\mathrm{N}_{\text {tot. }}-$ total nitrogen, $\mathrm{P}_{\text {tot. }}-$ total phosphorus, PAR - photosynthetically active radiation

The environmental factors affecting pondweeds in the selected categories of water bodies differed significantly in all their traits, except for the content of organic and mineral matter and of humic acid content in the sediment (Table 4). Furthermore, there was a significant difference between environmental factors at the sites of Potamogeton species and Stuckenia species, respectively (Table 5).

The heterogeneity of environmental conditions for pondweeds in the studied water bodies is presented in Fig. 2. The strongest positive correlation with the first PCA ordination axis is shown mainly by calcium concentration, water colour, water and sediment con- ductivity. Negative correlation with the first axis is demonstrated by water and sediment redox, and slightly weaker by oxygenation. The strongest correlation with the second axis is shown by water depth, and slightly less by sediment hydration, $\mathrm{CO}_{2}$ content, flow, content of $\mathrm{SiO}_{2}$ and $\mathrm{N}_{\text {tot. }}$

The occurrence of pondweeds in the lakes strongly correlated with the second PCA ordination axis. Positive correlation was shown by water depth, content of $\mathrm{CO}_{2}$, sediment $\mathrm{Ca}$ and organic matter, while negative correlation was demonstrated, primarily, by the flow and $\mathrm{SiO}_{2}$ content. Environmental factors for Potamogeton and Stuckenia in the lakes were similar. 
Table 5. Environmental factors for Potamogeton and Stuckenia in the waterbodies studied. Statistically significant differences at $\mathrm{p}<0.05$ are presented in bold type

\begin{tabular}{|c|c|c|c|c|c|c|c|c|c|c|}
\hline \multirow[b]{2}{*}{ Trait } & \multicolumn{4}{|c|}{$\begin{array}{c}\text { Potamogeton } \\
\mathrm{n}=836\end{array}$} & \multicolumn{4}{|c|}{$\begin{array}{c}\text { Stuckenia } \\
\mathrm{n}=276\end{array}$} & \multicolumn{2}{|c|}{$\begin{array}{c}\text { Mann-Whitney } \\
\text { U test }\end{array}$} \\
\hline & mean & SD & $\min$ & $\max$ & mean & SD & $\min$ & $\max$ & $\mathrm{Z}$ & $\mathrm{p}$ \\
\hline Depth [m] & 1.5 & 1.2 & 0 & 6.3 & 1.3 & 0.9 & 0.1 & 4.6 & 1.419 & 0.156 \\
\hline \multicolumn{11}{|c|}{ water traits } \\
\hline $\mathrm{pH}$ & 7.90 & 0.48 & 4.79 & 8.87 & 8.01 & 0.43 & 6.31 & 8.85 & -3.380 & $<0.001$ \\
\hline Conductivity $\left[\mu \mathrm{S} \mathrm{cm}^{-1}\right]$ & 314 & 257 & 34.5 & 1136 & 378 & 305 & 41.9 & 1136 & -3.734 & $<0.001$ \\
\hline Redox $[\mathrm{mV}]$ & 47 & 97 & -276 & 395 & 53 & 94 & -276 & 254 & -2.601 & 0.009 \\
\hline Colour [mg Pt dm $\left.{ }^{-3}\right]$ & 24 & 33 & 2 & 400 & 22 & 18 & 2 & 80 & 0.290 & 0.772 \\
\hline $\mathrm{HA}\left[\mathrm{mg} \mathrm{dm}^{-3}\right]$ & 3.6 & 4.1 & 0.3 & 45.1 & 3.3 & 2.1 & 0.7 & 9.4 & -1.678 & 0.093 \\
\hline $\mathrm{CO}_{2}\left[\mathrm{mg} \mathrm{dm}^{-3}\right]$ & 7.0 & 5.8 & 0.1 & 41.4 & 6.2 & 4.6 & 0.7 & 19.4 & 1.499 & 0.134 \\
\hline $\mathrm{CO}_{3}^{2-}\left[\mathrm{mg} \mathrm{CO}_{2} \mathrm{dm}^{-3}\right]$ & 8.8 & 9.2 & 0 & 62.9 & 7.0 & 7.6 & 0 & 28.6 & 2.795 & 0.005 \\
\hline $\mathrm{HCO}_{3}^{-}\left[\mathrm{mg} \mathrm{CO}_{2} \mathrm{dm}^{-3}\right]$ & 91.5 & 60.5 & 7.0 & 311.1 & 89.3 & 46.0 & 7.0 & 311.1 & -1.631 & 0.103 \\
\hline $\mathrm{Ca}^{2+}\left[\mathrm{mg} \mathrm{dm}^{-3}\right]$ & 46.9 & 24.6 & 1.0 & 131.5 & 54.8 & 27.6 & 3.4 & 131.5 & -4.322 & $<0.001$ \\
\hline $\mathrm{N}_{\text {tot. }}\left[\mathrm{mg} \mathrm{N} \mathrm{dm}^{-3}\right]$ & 1.56 & 1.16 & 0.30 & 5.27 & 1.44 & 1.08 & 0.38 & 5.27 & 1.933 & 0.053 \\
\hline $\mathrm{P}_{\text {tot. }}\left[\mathrm{mg} \mathrm{P} \mathrm{dm}^{-3}\right]$ & 0.34 & 0.23 & 0.02 & 1.47 & 0.29 & 0.19 & 0.03 & 1.22 & 2.574 & 0.010 \\
\hline $\mathrm{Cl}^{-}\left[\mathrm{mg} \mathrm{dm}^{-3}\right]$ & 19.6 & 98.3 & 0.5 & 2757 & 250.0 & 894.6 & 0.5 & 5121 & -2.930 & 0.003 \\
\hline PAR [\%] & 29.1 & 22.3 & 0.1 & 100 & 30.4 & 20.0 & 0.5 & 100 & -1.589 & 0.112 \\
\hline $\mathrm{O}_{2}[\%]$ & 102.0 & 29.2 & 7.6 & 161.2 & 107.7 & 17.8 & 12.3 & 147.5 & -1.684 & 0.092 \\
\hline Flow $\left[\mathrm{ms}^{-1}\right]$ & 0.06 & 0.13 & 0 & 0.64 & 0.07 & 0.15 & 0 & 0.64 & 0.381 & 0.703 \\
\hline \multicolumn{11}{|c|}{ sediment traits } \\
\hline $\mathrm{pH}$ & 7.10 & 0.37 & 5.10 & 8.24 & 7.19 & 0.30 & 6.49 & 7.98 & -2.782 & 0.005 \\
\hline Conductivity $\left[\mu \mathrm{S} \mathrm{cm}^{-1}\right]$ & 358.7 & 345.3 & 40.0 & 2150 & 291.2 & 186.0 & 41.9 & 879 & 0.603 & 0.547 \\
\hline Redox $[\mathrm{mV}]$ & -226 & 127 & -650 & 267 & -235 & 110 & -457 & 256 & 1.357 & 0.175 \\
\hline $\mathrm{Ca}^{2+}\left[\mathrm{mg} \mathrm{g}^{-1}\right.$ d.w. $]$ & 52.9 & 91.8 & 0.4 & 376.9 & 56.2 & 95.3 & 0.7 & 354.0 & -0.625 & 0.532 \\
\hline $\mathrm{HA}\left[\mathrm{mg} \mathrm{g}^{-1}\right.$ d.w. $]$ & 8.5 & 13.8 & 0.05 & 135.1 & 4.7 & 7.9 & 0.05 & 43.9 & 5.265 & $<0.001$ \\
\hline Hydration [\%] & 38.6 & 29.5 & 0.5 & 99.9 & 34.2 & 25.4 & 0.4 & 92.3 & 1.213 & 0.225 \\
\hline Organic matter [\%] & 10.3 & 15.2 & 0.2 & 92.8 & 6.1 & 8.6 & 0.3 & 38.9 & 3.826 & $<0.001$ \\
\hline Mineral matter [\%] & 89.3 & 16.3 & 7.2 & 99.8 & 93.2 & 11.5 & 38.1 & 99.7 & -3.755 & $<0.001$ \\
\hline $\mathrm{SiO}_{2}[\%]$ & 71.0 & 31.6 & 0.2 & 100 & 72.1 & 32.6 & 0.2 & 100 & 0.349 & 0.727 \\
\hline Fraction $>2 \mathrm{~mm}[\%]$ & 6.4 & 12.8 & 0.0 & 76.8 & 4.6 & 10.8 & 0.0 & 66.4 & 1.384 & 0.166 \\
\hline $1-2 \mathrm{~mm}[\%]$ & 3.6 & 4.3 & 0.0 & 20.2 & 2.9 & 4.1 & 0.0 & 20.2 & 1.788 & 0.074 \\
\hline $0.5-1 \mathrm{~mm}[\%]$ & 10.5 & 11.6 & 0.0 & 57.4 & 9.1 & 10.1 & 0.0 & 57.4 & 0.898 & 0.369 \\
\hline $0.25-0.5 \mathrm{~mm}[\%]$ & 23.5 & 18.4 & 0.0 & 65.2 & 24.7 & 20.2 & 0.0 & 65.2 & -0.891 & 0.373 \\
\hline $0.1-0.25 \mathrm{~mm}[\%]$ & 26.4 & 18.3 & 0.0 & 80.7 & 35.3 & 21.8 & 0.0 & 84.5 & -5.613 & $<0.001$ \\
\hline$<0.1 \mathrm{~mm}[\%]$ & 29.2 & 35.4 & 0.05 & 100 & 23.2 & 28.2 & 0.04 & 100 & 1.524 & 0.127 \\
\hline
\end{tabular}

Explanations: see Fig. 2

The occurrence of pondweeds in watercourses closely correlateed with the second ordination axis factors. Positive correlation was found with flow, $\mathrm{SiO}_{2}$ and $\mathrm{N}_{\text {tot. }}$ content, sediment $\mathrm{pH}$ and the $>0.25 \mathrm{~mm}$ fraction, and negative correlation with $<0.1 \mathrm{~mm}$, sediment hydration and depth. Compared with Potamogeton, Stuckenia occurred in shallower, faster-flowing waters, on less hydrated sediment of medium or large grain size with a small proportion of the smallest fraction $(<0.1 \mathrm{~mm})$.

The occurrence of pondweeds in estuaries correlated with the factors from the first PCA ordination axis. Environmental factors for Stuckenia correlated positively with calcium concentration, water colour, water conductivity and humic acid content, while showing negative correlation with water redox. Environmental 
Table 6. Environmental factors for Potamogeton and Stuckenia in the studied lakes. Statistically significant differences at $\mathrm{p}<0.05$ are presented in bold type

\begin{tabular}{|c|c|c|c|c|c|c|c|c|c|c|}
\hline \multirow[b]{2}{*}{ Trait } & \multicolumn{4}{|c|}{ Potamogeton } & \multicolumn{4}{|c|}{ Stuckenia } & \multicolumn{2}{|c|}{$\begin{array}{c}\text { Mann-Whitney } \\
\text { U test }\end{array}$} \\
\hline & mean & SD & $\min$ & $\max$ & mean & SD & $\min$ & $\max$ & Z & $\mathrm{p}$ \\
\hline Depth $[\mathrm{m}]$ & 1.81 & 1.3 & 0.00 & 6.30 & 1.57 & 1.1 & 0.10 & 4.60 & 1.299 & 0.194 \\
\hline \multicolumn{11}{|c|}{ water traits } \\
\hline $\mathrm{pH}$ & 7.94 & 0.5 & 4.79 & 8.87 & 8.04 & 0.3 & 6.31 & 8.72 & -2.008 & 0.045 \\
\hline Conductivity $\left[\mu \mathrm{S} \mathrm{cm}^{-1}\right]$ & 191 & 67 & 35 & 336 & 218 & 52 & 42 & 336 & -4098 & $<0.001$ \\
\hline Redox $[\mathrm{mV}]$ & 54 & 84 & -120 & 395 & 73 & 76 & -81 & 254 & -3.846 & $<0.001$ \\
\hline Colour [mg Pt dm $\left.{ }^{-3}\right]$ & 18 & 33 & 2 & 400 & 13 & 9 & 2 & 35 & 2.058 & 0.040 \\
\hline $\mathrm{HA}\left[\mathrm{mg} \mathrm{dm}^{-3}\right]$ & 2.8 & 4.3 & 0.3 & 45.1 & 2.4 & 1.4 & 0.7 & 7.2 & -1.200 & 0.230 \\
\hline $\mathrm{CO}_{2}\left[\mathrm{mg} \mathrm{dm}^{-3}\right]$ & 7.9 & 6.6 & 0.1 & 41.4 & 7.6 & 5.3 & 2.0 & 19.4 & -1.225 & 0.221 \\
\hline $\mathrm{CO}_{3}^{2-}\left[\mathrm{mg} \mathrm{CO}_{2} \mathrm{dm}^{-3}\right]$ & 9.6 & 10.3 & 0.0 & 62.9 & 8.7 & 8.8 & 0.0 & 28.6 & 0.983 & 0.326 \\
\hline $\mathrm{HCO}_{3}^{-}\left[\mathrm{mg} \mathrm{CO}_{2} \mathrm{dm}^{-3}\right]$ & 60.8 & 26.8 & 7.0 & 140.8 & 69.2 & 24.6 & 7.0 & 140.8 & -4.235 & $<0.001$ \\
\hline $\mathrm{Ca}^{2+}\left[\mathrm{mg} \mathrm{dm}^{-3}\right]$ & 34.4 & 13.4 & 1.0 & 69.2 & 40.2 & 8.9 & 3.4 & 68.1 & -5.297 & $<0.001$ \\
\hline $\mathrm{N}_{\text {tot }}\left[\mathrm{mg} \mathrm{N} \mathrm{dm}^{-3}\right]$ & 1.40 & 0.9 & 0.29 & 4.0 & 1.31 & 0.9 & 0.38 & 3.79 & 1.857 & 0.063 \\
\hline $\mathrm{P}_{\text {tot }}\left[\mathrm{mg} \mathrm{P} \mathrm{dm}^{-3}\right]$ & 0.25 & 0.2 & 0.02 & 1.34 & 0.20 & 0.1 & 0.03 & 1.22 & 2.705 & 0.007 \\
\hline $\mathrm{Cl}^{-}\left[\mathrm{mg} \mathrm{dm}^{-3}\right]$ & 5.9 & 5.7 & 0.5 & 50.3 & 7.3 & 6.7 & 0.5 & 50.3 & -2.806 & 0.005 \\
\hline $\operatorname{PAR}[\%]$ & 27.9 & 20.9 & 1.5 & 100.0 & 31.3 & 17.6 & 4.7 & 100.0 & -3.596 & $<0.001$ \\
\hline $\mathrm{O}_{2}[\%]$ & 110.8 & 20.1 & 12.3 & 161.2 & 112.2 & 11.8 & 12.3 & 136.1 & 0.077 & 0.938 \\
\hline Flow $\left[\mathrm{ms}^{-1}\right]$ & 0.00 & 0.0 & 0.00 & 0.00 & 0.00 & 0.0 & 0.00 & 0.00 & 0.000 & 1.000 \\
\hline \multicolumn{11}{|c|}{ sediment traits } \\
\hline $\mathrm{pH}$ & 7.04 & 0.4 & 5.10 & 8.24 & 7.16 & 0.3 & 6.46 & 7.76 & -3.459 & 0.001 \\
\hline Conductivity $\left[\mu \mathrm{S} \mathrm{cm}^{-1}\right]$ & 280 & 235 & 41 & 2150 & 278 & 168 & 42 & 879 & -0.896 & 0.370 \\
\hline Redox $[\mathrm{mV}]$ & -212 & 117 & -352 & 256 & -223 & 105 & -352 & 256 & 1.388 & 0.165 \\
\hline $\mathrm{Ca}^{2+}\left[\mathrm{mg} \mathrm{g}^{-1}\right.$ d.w. $]$ & 69.5 & 107.7 & 0.4 & 376.9 & 84.7 & 111.2 & 0.8 & 354.0 & -3.567 & $<0.001$ \\
\hline $\mathrm{HA}\left[\mathrm{mg} \mathrm{g}^{-1}\right.$ d.w. $]$ & 8.7 & 14.3 & 0.1 & 135.1 & 6.1 & 9.4 & 0.1 & 43.9 & 3.157 & 0.002 \\
\hline Hydration $[\%]$ & 39.8 & 31.4 & 0.5 & 99.9 & 38.8 & 30.5 & 0.4 & 92.3 & 0.727 & 0.467 \\
\hline Organic matter [\%] & 11.3 & 16.9 & 0.3 & 92.8 & 6.9 & 7.5 & 0.3 & 35.0 & 1.927 & 0.054 \\
\hline Mineral matter [\%] & 88.7 & 16.9 & 7.2 & 99.7 & 93.1 & 7.5 & 65.0 & 99.7 & -1.893 & 0.058 \\
\hline $\mathrm{SiO}_{2}[\%]$ & 67.4 & 35.2 & 0.2 & 100.0 & 62.7 & 36.5 & 0.2 & 100.0 & 2.181 & 0.029 \\
\hline Fraction $>2 \mathrm{~mm}[\%]$ & 5.8 & 12.5 & 0.0 & 76.8 & 5.0 & 11.9 & 0.0 & 66.4 & 0.841 & 0.400 \\
\hline $1-2 \mathrm{~mm}[\%]$ & 3.4 & 4.3 & 0.0 & 20.2 & 3.0 & 4.6 & 0.0 & 20.2 & 1.239 & 0.215 \\
\hline $0.5-1 \mathrm{~mm}[\%]$ & 8.7 & 9.9 & 0.0 & 37.3 & 7.8 & 9.7 & 0.0 & 36.2 & 0.870 & 0.384 \\
\hline $0.25-0.5 \mathrm{~mm}[\%]$ & 22.5 & 17.8 & 0.0 & 62.3 & 22.0 & 17.5 & 0.0 & 62.3 & 0.041 & 0.967 \\
\hline $0.1-0.25 \mathrm{~mm}[\%]$ & 28.4 & 18.8 & 0.0 & 80.7 & 35.3 & 21.5 & 0.0 & 83.4 & -3.238 & 0.001 \\
\hline$<0.1 \mathrm{~mm}[\%]$ & 30.9 & 35.5 & 0.1 & 100.0 & 26.7 & 30.2 & 0.0 & 100.0 & 0.711 & 0.477 \\
\hline
\end{tabular}

Explanations: see Fig. 2

factors for Potamogeton positively correlated mainly with sediment conductivity and the share of the $<0.1 \mathrm{~mm}$ fraction, while showing negative correlation with PAR and sediment redox (Fig. 2).

\subsection{Environmental factors in the lakes}

The lakes in which Potamogeton genus species occurred were diversified in terms of their hydrochemical properties (Table 6). Plants grew at a depth range from 0 to 6.3 metres $(1.8 \pm 1.3 \mathrm{~m})$ and a broad spectrum of water $\mathrm{pH}$ (4.8-8.9), conductivity $\left(34.5-336.0 \mu \mathrm{S} \mathrm{cm}^{-1}\right)$, and redox (-120-395 mV). Water was usually well oxygenated, but poorly photosynthetically irradiated, with a low concentration of humic acids, inorganic carbon, calcium and chlorides, but a fairly high concentration of nitrogen and phosphorus. Sediment was moderately hydrated; it contained little organic matter, but considerable amounts of inorganic matter $88.7 \pm 16.9 \%$ (66\% of silica, the rest being mostly calcium) and was chiefly composed of small fractions $<0.5 \mathrm{~mm}(>80 \%)$. Sediment $\mathrm{pH}(5.1-8.2)$, conductivity $\left(41.1-2150 \mu \mathrm{S} / \mathrm{cm}^{-1}\right)$ and redox $(-352-256 \mathrm{mV})$ showed significant variability.

Stuckenia species, compared with Potamogeton, grew in lakes of slightly higher reaction $(\mathrm{p}=0.045)$, conductivity $(p<0.001)$ and redox $(p<0.001)$. Concentrations of bicarbonates, calcium $(p<0.001)$ and chlorides were also higher ( $\mathrm{p}=0.005$; Table 6$)$, as was the PAR reaching plants $(\mathrm{p}<0.001)$. Total phosphorus 
Table 7. Environmental factors for Potamogeton and Stuckenia in the studied watercourses. Statistically significant differences at $\mathrm{p}<0.05$ are presented in bold type

\begin{tabular}{|c|c|c|c|c|c|c|c|c|c|c|}
\hline \multirow[b]{2}{*}{ Trait } & \multicolumn{4}{|c|}{ Potamogeton } & \multicolumn{4}{|c|}{ Stuckenia } & \multicolumn{2}{|c|}{$\begin{array}{l}\text { Mann-Whitney } \\
\text { U test }\end{array}$} \\
\hline & mean & SD & $\min$ & $\max$ & mean & SD & $\min$ & $\max$ & Z & $\mathrm{p}$ \\
\hline Depth [m] & 0.8 & 0.6 & 0.1 & 2.4 & 0.6 & 0.2 & 0.2 & 1.4 & -1.104 & 0.270 \\
\hline \multicolumn{11}{|c|}{ water traits } \\
\hline $\mathrm{pH}$ & 7.82 & 0.4 & 7.26 & 8.42 & 7.75 & 0.4 & 7.44 & 8.42 & 1.802 & 0.072 \\
\hline Conductivity $\left[\mu \mathrm{S} \mathrm{cm}^{-1}\right]$ & 289 & 104 & 189 & 870 & 272 & 37 & 189 & 308 & -1.460 & 0.144 \\
\hline Redox $[\mathrm{mV}]$ & 52 & 91 & -86 & 322 & 59 & 71 & -7 & 241 & -2.437 & 0.015 \\
\hline Colour [mg Pt dm ${ }^{-3}$ ] & 20 & 10 & 8 & 45 & 28 & 11 & 12 & 40 & -4.894 & $<0.001$ \\
\hline $\mathrm{HA}\left[\mathrm{mg} \mathrm{dm}{ }^{-3}\right]$ & 3.8 & 1.8 & 1.2 & 8.3 & 4.1 & 1.3 & 2.0 & 6.5 & -0.823 & 0.411 \\
\hline $\mathrm{CO}_{2}\left[\mathrm{mg} \mathrm{dm}^{-3}\right]$ & 5.8 & 4.3 & 0.7 & 24.2 & 2.8 & 1.3 & 1.3 & 5.7 & 6.078 & $<0.001$ \\
\hline $\mathrm{CO}_{3}^{2-}\left[\mathrm{mg} \mathrm{CO}_{2} \mathrm{dm}^{-3}\right]$ & 5.1 & 5.5 & 0.0 & 26.4 & 4.7 & 2.9 & 0.0 & 10.1 & -1.438 & 0.150 \\
\hline $\mathrm{HCO}_{3}^{-}\left[\mathrm{mg} \mathrm{CO}_{2} \mathrm{dm}^{-3}\right]$ & 111.7 & 45.3 & 59.0 & 286 & 95.1 & 21.3 & 59.0 & 118.8 & 1.797 & 0.072 \\
\hline $\mathrm{Ca}^{2+}\left[\mathrm{mg} \mathrm{dm}^{-3}\right]$ & 52.4 & 12.4 & 41.0 & 94.5 & 48.9 & 5.2 & 42.2 & 62.1 & 1.044 & 0.296 \\
\hline $\mathrm{N}_{\text {tot. }}\left[\mathrm{mg} \mathrm{N} \mathrm{dm}^{-3}\right]$ & 2.00 & 1.7 & 0.31 & 5.27 & 1.79 & 1.5 & 0.60 & 5.27 & -0.178 & 0.859 \\
\hline $\mathrm{P}_{\text {tot. }}\left[\mathrm{mg} \mathrm{P} \mathrm{dm}^{-3}\right]$ & 0.43 & 0.2 & 0.07 & 0.76 & 0.44 & 0.1 & 0.30 & 0.76 & 0.548 & 0.584 \\
\hline $\mathrm{Cl}^{-}\left[\mathrm{mg} \mathrm{dm}^{-3}\right]$ & 7.4 & 8.9 & 2.0 & 70.0 & 5.4 & 1.2 & 2.9 & 9.0 & 3.129 & 0.002 \\
\hline PAR [\%] & 42.4 & 23.2 & 0.4 & 100.0 & 36.8 & 14.4 & 13.2 & 74.0 & 2.160 & 0.038 \\
\hline $\mathrm{O}_{2}[\%]$ & 100.2 & 32.0 & 35.9 & 147.5 & 107.2 & 25.6 & 58.6 & 147.5 & -2.712 & 0.007 \\
\hline Flow $\left[\mathrm{ms}^{-1}\right]$ & 0.20 & 0.1 & 0.01 & 0.46 & 0.14 & 0.1 & 0.07 & 0.34 & 2.437 & 0.015 \\
\hline \multicolumn{11}{|c|}{ sediment traits } \\
\hline $\mathrm{pH}$ & 7.32 & 0.3 & 6.66 & 7.98 & 7.49 & 0.3 & 7.05 & 7.98 & -3.370 & 0.001 \\
\hline Conductivity $\left[\mu \mathrm{S} \mathrm{cm} \mathrm{cm}^{-1}\right]$ & 390 & 462 & 42 & 1435 & 132 & 87 & 52 & 364 & 4.153 & $<0.001$ \\
\hline Redox $[\mathrm{mV}]$ & -195 & 131 & -650 & 267 & -182 & 91 & -337 & 116 & -0.242 & 0.809 \\
\hline $\mathrm{Ca}^{2+}\left[\mathrm{mg} \mathrm{g}^{-1}\right.$ d.w. $]$ & 20.0 & 37.8 & 1.0 & 188.4 & 3.9 & 1.9 & 1.0 & 6.7 & 2.946 & 0.003 \\
\hline $\mathrm{HA}\left[\mathrm{mg} \mathrm{g}^{-1}\right.$ d.w. $]$ & 6.5 & 14.2 & 0.2 & 60.4 & 2.0 & 3.0 & 0.2 & 8.5 & 2.766 & 0.006 \\
\hline Hydration [\%] & 30.3 & 21.9 & 0.8 & 83.2 & 21.9 & 7.0 & 8.8 & 34.1 & 1.186 & 0.236 \\
\hline Organic matter [\%] & 7.9 & 12.5 & 0.3 & 38.9 & 7.1 & 13.9 & 0.3 & 38.9 & 2.245 & 0.025 \\
\hline Mineral matter $[\%]$ & 89.9 & 18.4 & 38.1 & 99.7 & 89.3 & 22.3 & 38.1 & 99.7 & -2.245 & 0.025 \\
\hline $\mathrm{SiO}_{2}[\%]$ & 78.1 & 24.3 & 32.4 & 98.4 & 85.4 & 21.4 & 37.3 & 98.3 & -2.404 & 0.016 \\
\hline Fraction $>2 \mathrm{~mm}[\%]$ & 11.3 & 15.9 & 0.0 & 62.4 & 5.5 & 11.2 & 0.00 & 62.4 & 2.681 & 0.007 \\
\hline $1-2 \mathrm{~mm}[\%]$ & 5.3 & 4.2 & 0.0 & 14.9 & 2.5 & 2.8 & 0.1 & 10.0 & 4.640 & $<0.001$ \\
\hline $0.5-1 \mathrm{~mm}[\%]$ & 17.3 & 13.0 & 0.0 & 57.4 & 14.8 & 11.2 & 1.0 & 57.4 & 1.629 & 0.103 \\
\hline $0.25-0.5 \mathrm{~mm}[\%]$ & 29.9 & 16.2 & 0.0 & 65.2 & 39.5 & 20.9 & 5.3 & 65.2 & -2.909 & 0.004 \\
\hline $0.1-0.25 \mathrm{~mm}[\%]$ & 24.4 & 16.3 & 0.0 & 52.9 & 33.8 & 26.0 & 3.7 & 84.5 & -2.766 & 0.006 \\
\hline$<0.1 \mathrm{~mm}[\%]$ & 11.5 & 21.7 & 0.1 & 100.0 & 3.4 & 3.7 & 0.3 & 8.9 & 2.828 & 0.005 \\
\hline
\end{tabular}

Explanations: see Fig. 2

was lower ( $\mathrm{p}=0.007)$, but highly variable $(0.03-1.22 \mathrm{mg}$ $\left.\mathrm{dm}^{-3}\right)$. Sediment was rich in mineral matter, with the $\mathrm{pH}$ slightly higher than in the case of Potamogeton $(p=0.001)$. The proportion of humic acids and silica was generally lower ( $p=0.002$ and $p=0.029$, respectively), the prevailing fraction being $0.1-0.25 \mathrm{~mm}$, while calcium content was higher $(\mathrm{p}<0.001$; Table 6$)$.

\subsection{Environmental factors in watercourses}

In the watercourses with Potamogeton, water was slightly alkaline ( $\mathrm{pH} 7.8 \pm 0.4)$, with moderately high conductivity and positive redox (Table 7). It was also well oxygenated, slightly coloured, relatively well photosynthetically irradiated, rich in nitrogen, phosphorus and inorganic carbon $\left(\mathrm{HCO}_{3}^{-}\right.$and $\left.\mathrm{CO}_{2}\right)$, but poor in chloride ions. The flow rate was moderate $\left(0.20 \pm 0.1 \mathrm{~m} \mathrm{~s}^{-1}\right)$, with a broad variation range $(0.01$ $\left.0.46 \mathrm{~m} \mathrm{~s}^{-1}\right)$. Sediment was rich in mineral matter, with a high proportion of silica and the prevailing fraction was fine grain, mostly $0.5-0.25 \mathrm{~mm}$. Calcium and humic acid concentration varied considerably (1.0-188.4 mg $\mathrm{Ca}^{2+} \mathrm{g}^{-1}$ d.w.; 0.2-60.4 $\mathrm{mg} \mathrm{C} \mathrm{g}^{-1}$ d.w. respectively).

At the sites with Stuckenia, water was more strongly coloured $(\mathrm{p}<0.001)$ than at the sites with Potamogeton; it was also poorer in carbon dioxide $(\mathrm{p}<0.001)$ and in chlorides ( $\mathrm{p}=0.002$; Table 7). Oxygen concentration in water was higher $(\mathrm{p}=0.007)$, while insolation and flow rate were lower ( $\mathrm{p}=0.038$ and $\mathrm{p}=0.015$, respectively). 
Table 8. Environmental factors for Potamogeton and Stuckenia in the studied estuaries. Statistically significant differences at $\mathrm{p}<0.05$ are presented in bold type

\begin{tabular}{|c|c|c|c|c|c|c|c|c|c|c|}
\hline \multirow[b]{2}{*}{ Trait } & \multicolumn{4}{|c|}{ Potamogeton } & \multicolumn{4}{|c|}{ Stuckenia } & \multicolumn{2}{|c|}{$\begin{array}{c}\text { Mann-Whitney } \\
\text { U test }\end{array}$} \\
\hline & mean & SD & $\min$ & $\max$ & mean & SD & $\min$ & $\max$ & $\mathrm{Z}$ & $\mathrm{p}$ \\
\hline Depth [m] & 1.11 & 0.6 & 2.50 & 0.30 & 0.96 & 0.4 & 1.60 & 0.30 & 1.319 & 0.187 \\
\hline \multicolumn{11}{|c|}{ water traits } \\
\hline $\mathrm{pH}$ & 7.85 & 0.5 & 6.91 & 8.74 & 8.19 & 0.6 & 6.91 & 8.85 & -4.547 & $<0.001$ \\
\hline Conductivity $\left[\mu \mathrm{S} \mathrm{cm}^{-1}\right]$ & 874 & 162 & 451 & 1136 & 970 & 130 & 666 & 1136 & -3.802 & $<0.001$ \\
\hline Redox $[\mathrm{mV}]$ & 13 & 141 & -276 & 255 & -12 & 129 & -276 & 178 & 1.004 & 0.315 \\
\hline Colour [mg Pt dm $\left.{ }^{-3}\right]$ & 58 & 32 & 15 & 160 & 42 & 24 & 15 & 80 & 3.230 & 0.001 \\
\hline $\mathrm{HA}\left[\mathrm{mg} \mathrm{dm}{ }^{-3}\right]$ & 6.8 & 3.7 & 2.3 & 17.1 & 5.4 & 2.4 & 2.2 & 9.4 & 1.789 & 0.074 \\
\hline $\mathrm{CO}_{2}\left[\mathrm{mg} \mathrm{dm}^{-3}\right]$ & 5.3 & 3.4 & 0.7 & 11.4 & 4.7 & 1.6 & 0.7 & 8.4 & 0.648 & 0.517 \\
\hline $\mathrm{CO}_{3}^{2-}\left[\mathrm{mg} \mathrm{CO}_{2} \mathrm{dm}^{-3}\right]$ & 10.4 & 5.9 & 0.0 & 18.5 & 4.2 & 4.9 & 0.0 & 17.6 & 6.269 & $<0.001$ \\
\hline $\mathrm{HCO}_{3}^{-}\left[\mathrm{mg} \mathrm{CO}_{2} \mathrm{dm}^{-3}\right]$ & 197.2 & 52.0 & 92.4 & 311.1 & 145.4 & 63.5 & 92.4 & 311.1 & 6.166 & $<0.001$ \\
\hline $\mathrm{Ca}^{2+}\left[\mathrm{mg} \mathrm{dm}^{-3}\right]$ & 93.1 & 14.2 & 78.8 & 131.5 & 105.1 & 18.6 & 82.4 & 131.5 & -4.818 & $<0.001$ \\
\hline $\mathrm{N}_{\text {tot. }}\left[\mathrm{mg} \mathrm{N} \mathrm{dm}^{-3}\right]$ & 1.61 & 0.9 & 0.51 & 4.82 & 1.50 & 1.1 & 0.51 & 3.80 & 1.468 & 0.142 \\
\hline $\mathrm{P}_{\text {tot. }}\left[\mathrm{mg} \mathrm{P} \mathrm{dm}^{-3}\right]$ & 0.55 & 0.2 & 0.16 & 1.47 & 0.43 & 0.2 & 0.13 & 0.78 & 2.824 & 0.005 \\
\hline $\mathrm{Cl}^{-}\left[\mathrm{mg} \mathrm{dm}^{-3}\right]$ & 95.2 & 240 & 47.6 & 2757 & 1227 & 1692 & 47.6 & 5121 & -6.496 & $<0.001$ \\
\hline PAR [\%] & 16.3 & 17.4 & 0.1 & 100.0 & 21.7 & 27.3 & 0.5 & 100.0 & -1.240 & 0.215 \\
\hline $\mathrm{O}_{2}[\%]$ & 66.4 & 30.8 & 7.6 & 117.9 & 94.5 & 18.1 & 45.6 & 117.9 & -5.742 & $<0.001$ \\
\hline Flow $\left[\mathrm{ms}^{-1}\right]$ & 0.11 & 0.2 & 0.00 & 0.64 & 0.22 & 0.3 & 0.00 & 0.64 & 0.077 & 0.939 \\
\hline \multicolumn{11}{|c|}{ sediment traits } \\
\hline $\mathrm{pH}$ & 7.08 & 0.3 & 6.47 & 7.52 & 7.03 & 0.2 & 6.54 & 7.44 & 0.514 & 0.608 \\
\hline Conductivity $\left[\mu \mathrm{S} \mathrm{cm}^{-1}\right]$ & 653 & 393 & 40 & 1428 & 479 & 141 & 192 & 640 & 2.321 & 0.020 \\
\hline Redox $[\mathrm{mV}]$ & -326 & 115 & -460 & 33 & -316 & 101 & -457 & -33 & -1.038 & 0.299 \\
\hline $\mathrm{Ca}^{2+}\left[\mathrm{mg} \mathrm{g}^{-1}\right.$ d.w. $]$ & 26.5 & 32.5 & 1.4 & 153.0 & 15.2 & 23.5 & 0.7 & 67.7 & 3.814 & $<0.001$ \\
\hline $\mathrm{HA}\left[\mathrm{mg} \mathrm{g}^{-1}\right.$ d.w.] & 10.4 & 10.4 & 0.1 & 33.1 & 2.5 & 3.5 & 0.2 & 16.0 & 3.944 & $<0.001$ \\
\hline Hydration [\%] & 44.8 & 27.9 & 13.1 & 92.7 & 30.7 & 10.1 & 14.7 & 49.7 & 1.858 & 0.063 \\
\hline Organic matter [\%] & 8.8 & 8.7 & 0.2 & 28.2 & 2.4 & 2.2 & 0.3 & 10.4 & 3.564 & $<0.001$ \\
\hline Mineral matter [\%] & 91.2 & 8.7 & 71.9 & 99.8 & 97.6 & 2.2 & 89.6 & 99.7 & -3.564 & $<0.001$ \\
\hline $\mathrm{SiO}_{2}[\%]$ & 76.7 & 19.7 & 29.8 & 98.6 & 89.5 & 6.4 & 77.7 & 98.1 & -3.330 & $<0.001$ \\
\hline Fraction $>2 \mathrm{~mm}[\%]$ & 2.0 & 5.2 & 0.0 & 19.6 & 2.3 & 5.3 & 0.0 & 18.7 & -2.023 & 0.043 \\
\hline $1-2 \mathrm{~mm}[\%]$ & 2.5 & 4.0 & 0.0 & 13.5 & 2.9 & 3.6 & 0.0 & 11.7 & -2.229 & 0.026 \\
\hline $0.5-1 \mathrm{~mm}[\%]$ & 9.0 & 13.0 & 0.0 & 43.6 & 7.5 & 8.4 & 0.2 & 29.4 & -1.815 & 0.069 \\
\hline $0.25-0.5 \mathrm{~mm}[\%]$ & 19.0 & 21.6 & 0.0 & 64.5 & 19.1 & 20.9 & 0.4 & 64.5 & -1.470 & 0.142 \\
\hline $0.1-0.25 \mathrm{~mm}[\%]$ & 20.5 & 17.1 & 0.0 & 61.5 & 37.2 & 19.6 & 15.4 & 68.9 & -5.464 & $<0.001$ \\
\hline$<0.1 \mathrm{~mm}[\%]$ & 46.0 & 40.0 & 0.1 & 100.0 & 30.4 & 26.3 & 0.3 & 81.0 & 2.081 & $\mathbf{0 . 0 3 7}$ \\
\hline
\end{tabular}

Explanations: see Fig. 2

Sediment was mostly mineralised, with a higher proportion of silica $(\mathrm{p}=0.016)$, medium- or fine grained $(0.5$ 0.25 and $0.25-0.1 \mathrm{~mm}$ ) and very low content of calcium $(\mathrm{p}=0.003)$ and of humic acids $(\mathrm{p}=0.006)$. The reaction was higher $(\mathrm{pH} 7.5 \pm 0.3 ; \mathrm{p}=0.001)$ and conductivity was much lower $(\mathrm{p}<0.001$; Table 7$)$.

\subsection{Environmental factors in estuaries}

In the estuaries with Potamogeton, water was alkaline, strongly coloured, with high conductivity caused by the considerable concentration of chlorides, carbonates, bicarbonates and calcium (Table 8). Water was rich in phosphorus, but poorly photosynthetically irradiated and oxygenated. Sediment was mineralised, with a high silica content and the prevalence of fine and very fine grain $(<0.1 \mathrm{~mm}$ and $0.1-0.25 \mathrm{~mm})$. Calcium compound content was relatively low, while humic acid content exhibited great variability (0.1-33.1 $\mathrm{mg} \mathrm{C} \mathrm{g}^{-1} \mathrm{~d}$.w.).

Estuaries with Stuckenia were characterised, primarily, by a very high chloride content $(\mathrm{p}<0.001)$ and its great variability $\left(47.6-5121.4 \mathrm{mg} \mathrm{Cl}^{-} \mathrm{dm}^{-3}\right)$. The reaction was higher than in the case of Potamogeton $(\mathrm{p}<0.001)$, so was conductivity $(\mathrm{p}<0.001)$ and calcium concentration $(p<0.001)$, while phosphorus concentration was lower $(\mathrm{p}=0.005)$. Water was very strongly oxygenated and slightly coloured ( $\mathrm{p}=0.001$; Table 8 ) allowing more light to reach plants $(\mathrm{p}<0.001)$ than in the water bodies with Potamogeton. Sediment was mineralised and, as 


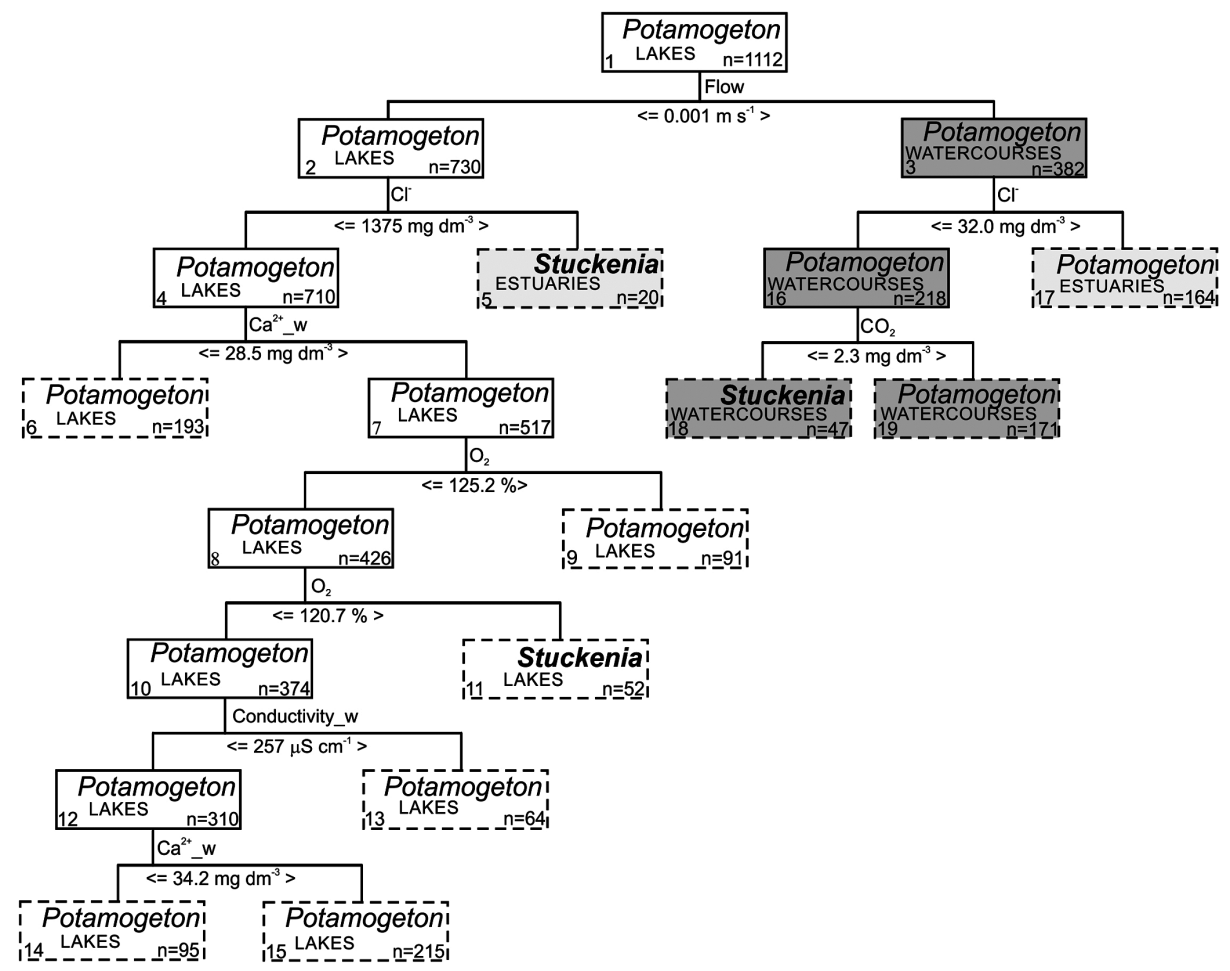

Fig. 3. Empirical model of Potamogeton and Stuckenia dependence on C\&RT-ranked environmental factors in the studied lakes, watercourses and estuaries

in the case of sites with Potamogeton, fine and very fine grain fractions prevailed, with a slightly higher share of silica $(p<0.001)$. Sediment conductivity was lower $(\mathrm{p}=0.02)$ as was the case with calcium and humic acid content $(\mathrm{p}<0.001$; Table 8$)$.

\subsection{The rank of environmental factors}

The dependence of Potamogeton and Stuckenia distribution on environmental factors in particular waterbody types is presented in an empirical model (Fig. 3), designed according to the Classification and Regression Trees (C\&RT) method. Two main branches of the model are shaped by water flow.

The left branch of the empirical model (Fig. 3) illustrates the environmental factors in the lakes, with low chloride concentration in water. This is where mainly Potamogeton communities developed. Differences within this group of water bodies were chiefly related to calcium and chloride concentration in water as well as its conductivity and oxygenation. Stuckenia communities were mainly associated with chloride-rich waters in estuaries (above $1375 \mathrm{mg} \mathrm{dm}^{-3}$ ) and with lakes in which water was well oxygenated (120.7-125.2\%).

The right branch of the model (Fig. 3) shows the environmental factors in water bodies of considerable flow rate, i.e., in watercourses, but also in some of the estuaries, where chloride concentration was higher than $32 \mathrm{mg} \mathrm{dm}^{-3}$. Here, it was mainly communities with Potamogeton which developed. Where chloride concentration was lower than $32 \mathrm{mg} \mathrm{dm}^{-3}$, and free carbon dioxide was below $2.3 \mathrm{mg} \mathrm{dm}^{-3}$, communities with Stuckenia pectinata developed.

The C\&RT model indicates that calcium concentration in water was the main environmental feature (validity coefficient $=1$ ) controlling pondweed distribution in the water bodies studied and affecting the mineral content of water. Predictive validity of environmental factors in this model indicates that equally important were: water conductivity (validity coefficient $=0.98$ ), concentration of $\mathrm{Cl}^{-}(0.93)$ and $\mathrm{CO}_{2}(0.83)$, water colour and flow (0.69 and 0.68 respectively; Fig. 4). It should be noted that the traits connected with water oxygenation and sediment redox are statistically significant in the model. The results may indicate that with the plant group of such diversity, other traits of environment may affect the distribution of particular species. The rank of other environmental factors in the model is much lower $(<0.5)$.

\section{Discussion}

Most of the pondweeds (Potamogetonaceae) in Pomerania are found in neutral or slightly alkaline water rich in calcium and bicarbonates. Similar observations have been made in North America (Ogden 1943; Moyle 1945; Hellquist 1980; Pip 1987), Japan (Kadono 1982) and elsewhere in Europe (Wiegleb 1984). The conducted studies demonstrated concentration of $\mathrm{Ca}$, water 


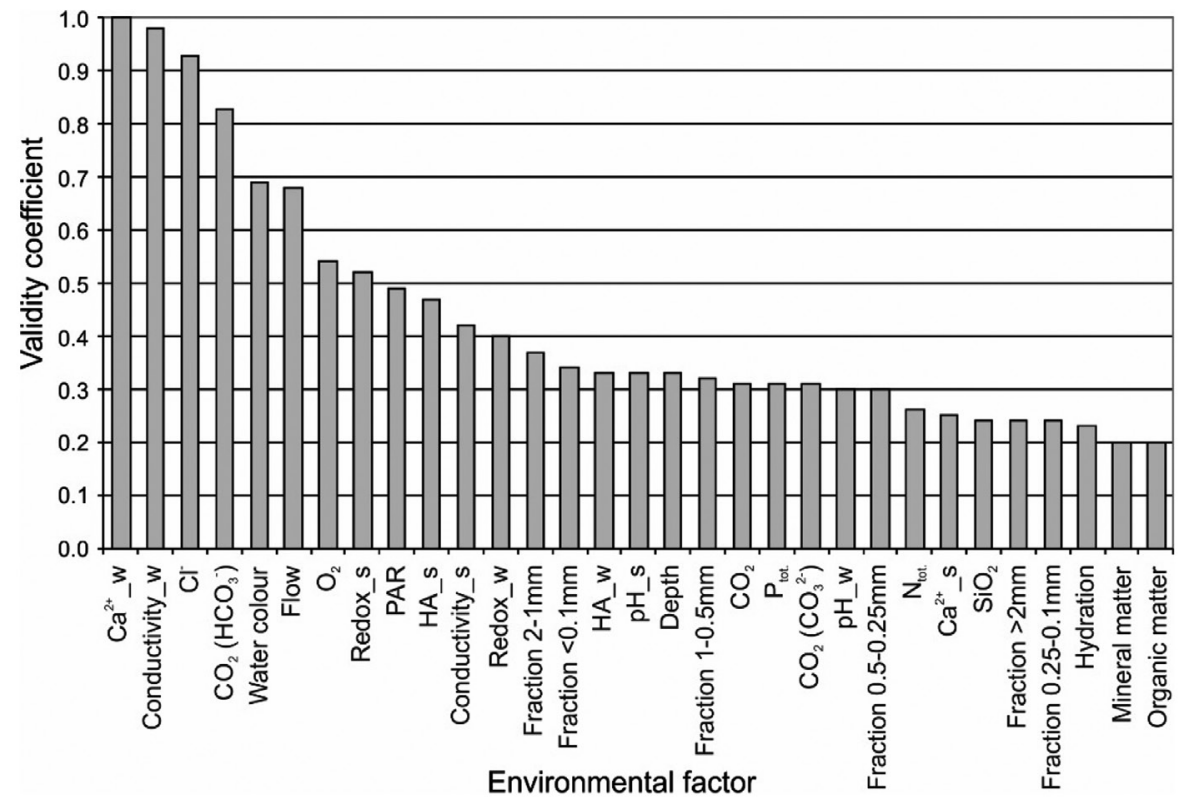

Fig. 4. Predictive validity of environmental factors in C\&RT model

conductivity, concentration of $\mathrm{Cl}^{-}$and $\mathrm{CO}_{2}$, water colour and flow to be the highest rank environmental factors for pondweeds (see Fig. 4). It should be stressed that the empirical model presented with the Classification and Regression Tree (C\&RT; Cf. Fig. 3) identifies water flow as the main factor determining pondweed occurrence in particular kinds of water bodies (lakes, watercourses and estuaries), making them the main branches of the classification tree. The remaining characteristics related to water mineral content, like the concentration of calcium, chlorides and carbonates, have an impact on waterbody differentiation within the particular branches of the tree.

The Potamogeton occurrence model, accounting for flow and $\mathrm{Ca}$ concentration only, is a source of new, detailed information (Fig. 5). Pondweed environmental factors in lakes, compared with other water bodies, are low concentration of calcium and the absence of water flow. In watercourses, pondweeds occurred in waters of considerable flow rate and only slightly higher calcium concentration than in lakes. In estuaries, pondweeds preferred waters with the highest calcium concentration and

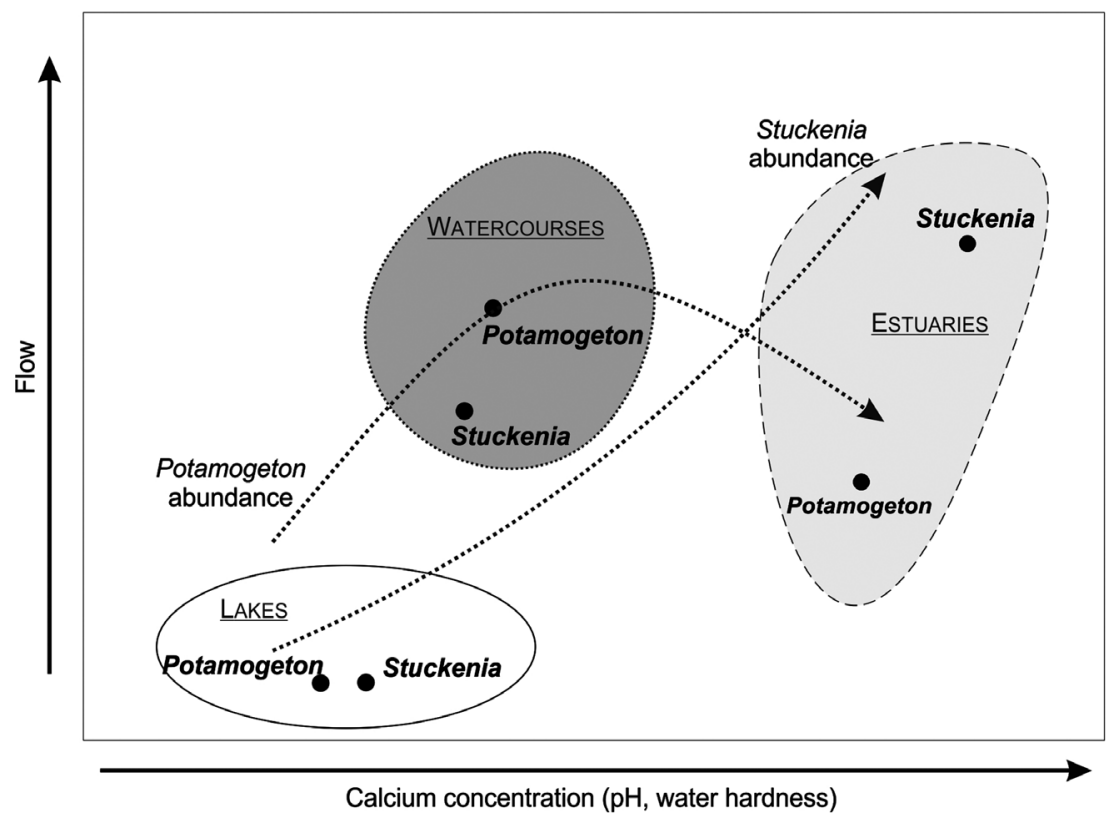

Fig. 5. Conceptual model for Potamogeton and Stuckenia in the studied lakes, watercourses and estuaries 


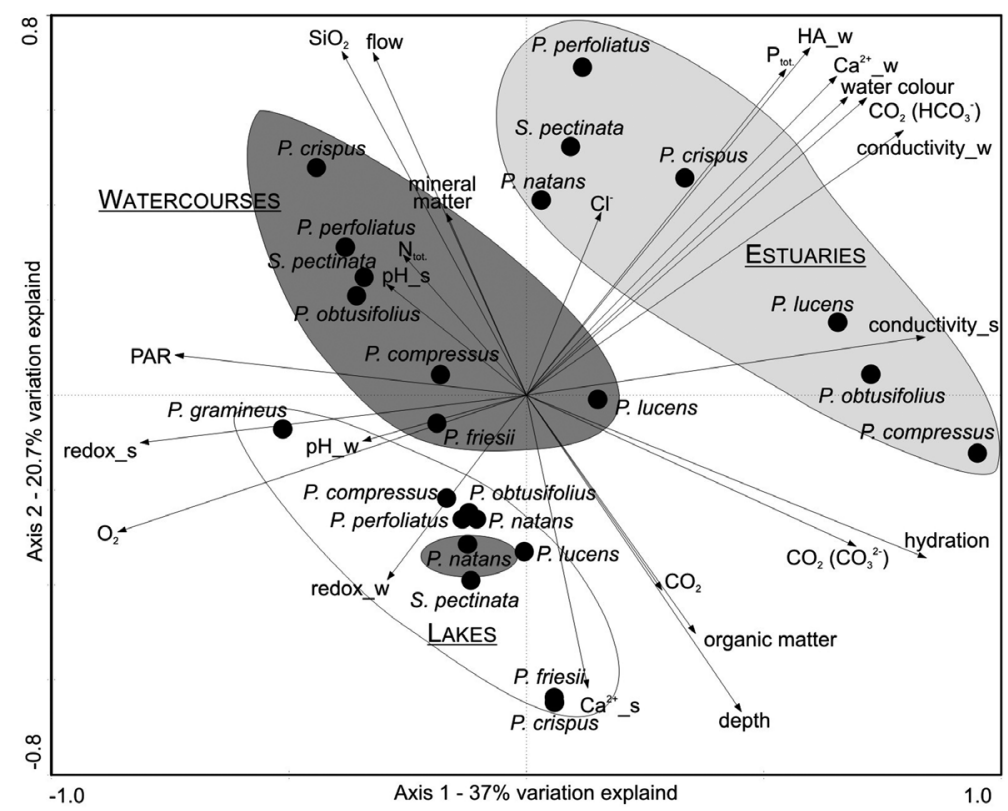

Fig. 6. Model of the relationship between the occurrence of most common pondweed species and PCA-ranked environmental factors in the studied lakes, watercourses and estuaries

Explanations: see Fig. 2

\begin{tabular}{lccccc}
\hline \multicolumn{7}{c}{ Summary of PCA analysis } & & & \\
\hline Axes & 1 & 2 & 3 & 4 & Total inertia \\
Eigenvalues: & 0.370 & 0.207 & 0.081 & 0.074 & 1.000 \\
Cumulative percentage variance of species data: & 37.0 & 57.7 & 65.8 & 73.2 & \\
Sum of all eigenvalues & & & & & 1.000 \\
\hline
\end{tabular}

varying flow. In lakes, Potamogeton showed a preference for lower calcium concentration than Stuckenia, in watercourses were connected with faster flow, while in estuaries - occurred in lower flow waters than Stuckenia. In lakes and watercourses, species of the Potamogeton genus were more abundant than Stuckenia, contrary to estuaries in which Stuckenia were more plentiful than Potamogeton. The model demonstrates the growing abundance of Stuckenia as water flow and calcium concentration grow (Fig. 5). It should be remembered that in the water bodies under examination, Stuckenia genus is represented only by Stuckenia pectinata; in lakes - also by Stuckenia filiformis. S. pectinata is a species of a broad range of environmental factors and is present in all kinds of water bodies. In their studies, both Hellquist (1980) and Kadono (1982) found the species to be distinctive and made it to be a separate group marked by high alkalinity and high content of bicarbonates and calcium. Chloride concentration in water bodies studied by Kadono (1982) in Japan varied from 16.9 to $4830 \mathrm{mg} \mathrm{dm}^{-3}$. The variability was even higher in Pomerania, ranging between 0.48 and $5121 \mathrm{mg} \mathrm{dm}^{-3}$. Such great environmental adaptability is the result of high phenotypic plasticity of S. pectinata (Kaplan 2002; Pilon \& Santamaria 2002; Santamaria et al. 2003).
Environmental factors affecting pondweeds in water bodies were diverse, mainly because of the large number of Potamogeton genus species and their different habitat requirements. They were also affected by a multitude of water and sediment traits (Fig. 6) in particular kinds of the water body.

In lakes, the heterogeneity of pondweed environmental conditions was largely dependent on water depth and the resulting water and sediment traits, with a lesser impact of water and sediment oxygenation and redox potential (Cf. Fig. 6). P. gramineus could be found in shallow and well photosynthetically irradiated lake areas. It only occurred in non-running waters and showed a preference for water bodies of moderate alkalinity and mineral content. Similar regularities were observed by researchers in the USA (Moyle 1945; Spence 1967; Hellquist 1980), Japan (Kadono 1982) and Estonia (Mäemets et al. 2010). Like in the area studied here, the species showed a preference for shallow zone of lake areas. Consequently, it can also survive periods outside the water body when the water table is low. A similar adaptability was shown by another species - P. polygonifolius. It also occurred in shallow lake areas, but of completely different physico-chemical properties than $P$. gramineus. It was recorded in only 
one site in Pomerania, in a humic lake situated in the coastal strip. Its water was highly acidic ( $\mathrm{pH} 4.79)$ and extremely poor in minerals $\left(48.9 \mu \mathrm{S} \mathrm{cm}^{-1}\right)$. In other sites in Poland (Lower Silesia) and in Germany (Saxony), the species occurred also in low $\mathrm{pH}$ peatbogs, but of considerably higher conductivity and calcium content (Zalewska-Gałosz et al. 2012). Spence (1972) placed the species in the macrophyte group, incapable of using bicarbonate ions for photosynthesis. A similar observation was made by Kadono (1980) concerning P. fryeri, which requires free $\mathrm{CO}_{2}$ in the water to grow. P. crispus and $P$. perfoliatus, on the other hand, are adapted to use carbon from bicarbonates for photosynthesis, so they can be found in many water bodies, particularly when water is highly alkaline and rich in bicarbonates and calcium. In the lakes studied here, $P$. crispus like $P$. friesii occurred in deep zones on the sediments rich in calcium and organic matter.

Pondweed environmental factors in watercourses were diversified, primarily, by high flow rate, resulting in high silica content and a significant proportion of coarse and medium grain sediment size. Preference for fast-flowing watercourses rich in silica was shown by $P$. crispus, while slow-flowing ones were preferred by $P$. compressus, $P$. friesii and $P$. lucens. $P$. natans deserves special attention, as it was the only species to occupy habitats of nearly identical properties, both in watercourses and in lakes (Cf. Fig. 6). Very rare species occurring in watercourses only comprised $P$. nodosus and $P$. $\times$ sparganifolius. The former can be found in water bodies of high and low alkalinity alike and is regarded as highly adaptable (New England: Hellquist 1980). However, the specimens from waters poor in bicarbonates were infertile. The observations were confirmed by Moor \& Clarkson (1967) and by Moyle (1945) in a different part of the United States and by Clapham et al. (1962) on the British Isles. In Pomerania, the species occurred in one highly-alkaline watercourse only and its specimens were fertile.
Pondweed environmental factors in estuaries may differ according to flow, sediment hydration, the amount of humic acids and sediment fraction of less than $0.1 \mathrm{~mm}$, but to a certain degree, also to the presence of minerals in water (conductivity, calcium concentration, chlorides and others). Estuaries of considerable flow rate and rich in chlorides, calcium and humic acids were dominated by Potamogeton perfoliatus and Stuckenia pectinata, while estuaries with highly hydrated sediment, rich in humic acids and fraction of less than $0.1 \mathrm{~mm}$ were preferred by $P$. lucens and P. obtusifolius, and, especially, by P. compressus (Fig. 6). P. crispus, in Pomerania and in Japan alike (Kadono 1982), shows a preference for waters rich in calcium and bicarbonates and for saltwater bodies. Moreover, Hellquist (1980) in New England as well as Moyle (1945) and McCombie \& Wile (1971) observed a significant correlation between the presence of the species and high biogenic content.

This was not observed in water bodies of northwestern Poland; however, the species was found to occupy waters of considerable flow rate - both in rivers and estuaries - which indicates its great adaptability.

Acknowledgments. The authors are deeply grateful to Professor Józef Szmeja for helpful comments received while this article was being written. This manuscript is based on the results obtained in research financed from the Department of Plant Ecology (DS-530-L145-D245-18).

\section{Autor Contributions}

Research concept and desing: Marek Merdalski, Krzysztof Banaś

Acquisition and/or assembly of data: Marek Merdalski, Krzysztof Banaś

Data analysis and interpretation: Marek Merdalski, Krzysztof Banaś

Drafting the article: Marek Merdalski, Krzysztof Banaś, Rafał Ronowski

Critical revision: Marek Merdalski, Krzysztof Banaś, Rafał Ronowski

Final approval: Marek Merdalski, Krzysztof Banaś, Rafał Ronowski

\section{References}

Afranowicz R. 2007. Ginące i zagrożone rośliny wodne na Żuławach Wiślanych w świetle dotychczasowych badań. Fragm. Flor. Geobot. Polonica 14(2): 319-335.

Aston H. I. 1973. Aquatic Plants of Australia. Melbourne University Press, Melbourne.

Austin M. P. 2007. Species Distribution Models and Ecological Theory: a Critical Assessment and Some Possible New Approaches. Ecol. Modell. 200: 1-19.

Bell P. A. 1999. The Impact of Sample Rotation Patterns and Composite Estimation on Survey Outcomes. Canberra: Australian Bureau of Statistics.

Bobrov A. A. \& Chemeris E. V. 2009. Pondweeds (Potamogeton, Potamogetonaceae) in River Ecosystems in the North of European Russia. Dokl. Biol. Sci. 425: $167-170$.

Börner C. 1912. Botanisch-systematische Notizen. Abh. Naturwiss. Vereine Bremen 21: 245-282.

Brux H., Herr W., Todeskino D. \& Wigleb G. 1988. A study on floristic structure and dynamics of communities with Potamogeton alpinus BALBIS in water bodies of the norther part of Federal Republic of Germany. Aquat. Bot. 32: 23-44.

Chambers, P. A. Prepas E. E, Hamilton H. R. \& Bothwell M. L. 1991. Current velocity and its effects on aquatic macrophytes in flowing waters. Ecol. Appl. 1: 249257. 
Chmara R., Banaś K. \& Szmeja J. 2015. Changes in the structural and functional diversity of macrophyte communities along an acidity gradient in softwater lakes. Flora 216: 57-64.

Chmara R. \& BociąG K. 2007. Rozmieszczenie rdestnic (Potamogeton; Potamogetonaceae) na Równinie Charzykowskiej (NW Polska). Fragm. Flor. Geobot. Polonica 14(2): 311-318.

Clapham A. R., Tutin I. G. \& Warburg E. F. 1962. Flora of the Brithis Isles. 2 ed. 1269 pp. Cambridge Univ. Press, Cambridge.

Dandy J. E. 1937. The genus Potamogeton L. in tropical Africa. J. Linn. Soc. Bot. 50: 507-540.

Eaton A. D., Clesceri L. S., Rice E. W. \& Greenberg A. E. 2005. Standard methods for the examination of water and wastewater. Washington: American Public Health Association.

Fernald M. L. 1932. The linear-leaved North America species of Potamogeton section Axillares. Mem. Amer. Acad. Arts. N. Ser. 17: 1-183.

GAFNy S. \& GAsith A. 1999. Spatially and temporally sporadic appearance of macrophytes in the littoral zone of Lake Kinneret, Israel. Taking advantage of a window of opportunity. Aquat. Bot. 62: 249-267.

GórniaK A. 1996. Substancje humusowe i ich rola w funkcjonowaniu ekosystemów słodkowodnych. Dział Wydawnictw Filii Uniwersytetu Warszawskiego, Białystok.

Hagström J. O. 1916. Critical researches on the Potamogetons. Kongl. Svenska Vetenskapsakad. Handl. 55: 1-281.

Haynes R. R. 1974. A revision of North American Potamogeton subsecyion Pusilli (Potamogetonaceae). Rhodora 76: 564-649.

Hastie T., Tibshirani R. \& Friedman J. 2001. The Elements of Statistical Learning; Data Mining, Inference and Prediction. New York: Springer Verlag.

Haynes R. R. \& Hellquist C. B. 2000. Potamogetonaceae DUMORTIER. In Flora of North America Editorial Committee (ed.) Flora of North America: north of Mexico 22. 47-74 pp. Oxford University Press, New York.

Haynes R. R., Les D. H. \& Kráol M. 1998. Two new combinations in Stuckenia, the correct name for Coleogeton (Potamogetonaceae). Novon 8:241.

Hellquist C. B. 1980. Correlation of Alkalinity and the Distribution of Potamogeton in New England. Rhodora 82: 331-344.

Hermanowicz W., Dożańska w., Dojlido J. \& Kozierowski B. 1999. Fizyko-chemiczne badania wody i ścieków. 555 pp. Arkady, Warszawa (in Polish).

Holub J. 1984. Some new nomenclatural Combinations I. Folia Geobot. Phytotax. 19(2): 213-215.

Kadono Y. 1980. Photosynthetic Carbon Sources in some Potamogeton Species. Bot. Mag. Tokyo 93: 185-194.

Kadono Y. 1982. Distribution and Habitat of Japanese Potamogeton. Bot. Mag. Tokyo 95: 63-76.

Kaplan Z. 2002. Phenotypic Plasticity in Potamogeton (Potamogetonaceae). Folia Geobot. 37: 141-170.

Kaplan Z. 2005. Neotypification of Potamogeton $\times$ fluitans Roth and the distribution of this hybrid. Taxon 54(3): 822-826.
KaPlan Z. 2008. A Taxonomic Revision of Stuckenia (Potamogetonaceae) in Asia, with Notes on the Diversity and Variation of the Genus on a Worldwide Scale. Folia Geobot. 43: 159-234.

Kaplan Z. 2010. Hybridization of Potamogeton Species in The Czech Republic - Diversity, Distribution, Temporal Trends and Habitat Preferences. Preslia 82: 261-287.

Kaplan Z. \& Symoens J.-J. 2005. Taxonomy, Distribution and Nomenclature of Three Confused Broad-leaved Potamogeton Species Occurring in Africa and on Surrounding Islands. Bot. J. of the Linn. Soc. 148: 329-357.

Kashina L. I. [= Kaschina L. I.] 1988. Semeistvo 24. Potamogetonaceae - Rdestovye. In: I. M. KRASNOBOROv (ed.). Flora Sibiri, Lycopodiaceae - Hydrocharitaceae, p. 93-105, 165-176. Nauka, Novosibirsk (in Russian).

Lehmann A., Castella E. \& Lachavanne J. B. 1997. Morphological traits and spatial heterogeneity of aquatic plants along sediment and depth gradients, Lake Geneva, Switzerland. Aquat. Bot. 55: 281-299.

Les D. H. \& Haynes R. R. 1996. Coleogeton (Potamogetonaceae), a new genus of pondweeds. Novon 6: 389-391.

Mądalski J. 1977. Atlas flory Polskiej i Ziem Ościennych - Florae Polonicae terrarumque adiacentium iconographia. 2(1). PWN, Warszawa, Wrocław, Kraków (in Polish).

Madsen J. D., Chambers P. A., James W. F., Koch E. W. \& Westlake D. F. 2001. The interaction between water movement, sediment dynamics and submersed macrophytes. Hydrobiologia 444: 71-84.

Mäemets A.A. 1979. Rod 2. Rdest - Potamogeton L. In: A. A. FEDOROw (ed.) Flora evropeiskoi chasti SSSR. vol. 4, pp. 176-192. Nauka, Leningrad (in Russian).

Mäemets A. A. 1984. 6. Potamogetonaceae Dumort In: K. Eichwald, E. KukK, V. Kuusk, E. Lellep, A. Mäemets, H. Rebassoo, R. Sander, S. Talts, T. Trei \& L. Viljasoo (eds.). Eesti NSV Floora, vol. 9, pp. 46-139. Valgus, Tallinn.

Mäemets H., Palmik K., Haldna M., Sudnitsyna D. \& MelNIK M. 2010. Eutrophication and Macrophyte Species Richness in the Large Shallow North-European Lake Peipsi. Aquat. Bot. 92: 273-280.

McCombie A. M. \& Wile I. 1971. Ecology of Aquatic Vascular Plants in Southern Ontario Impoundments. Weed Sci. 19: 225-228.

Miki S. 1937. The water phanerogams in Japan with special reference to those of the province Yamashiro. Raports on the historical remains, scenic places and natural monuments in Kyoto Pref. 18: 1-127 [in Japanese].

Moore J. A. \& Clarkson R. B. 1967. Physical and Chemical Factors Affecting Vascular Aquatic Plants in some Acid Stream Drainage Areas of the Monongahela River. Proc. W. Va. Acad. Sci. 39: 83-89.

Moore T. R. 1985. The Spectrophotometric Determination of Dissolved Organic Carbon in Peat Water. Soil Sci. Soc. Ann. J. 49: 1590-1592.

Moore T. R. 1987. An Assessment of a Simple Spectrophotometric Method for the Determination of Dissolved Organic Carbon in Freshwaters. New Zeal. J. Mar. Fresh. Res. 21: 585-589. 
Moyle J. B. 1945. Some Chemical Factors Influencing the Distribution of Aquatic Plants in Minnesota. Am. Midl. Nat. 34: 402-420.

Murphy K. J., Dickinson G., Thomaz S. M., Bini L. M., Dick K., Greaves K., Kennedy M., Livingstone S., McFerran H., Milne J., Oldroyd J. \& Wingfield R. 2003. Aquatic plant communities and predictors of diversity in a sub-tropical river floodplain: the Upper Rio Parana, Brazil. Aquat. Bot. 77: 257-276.

Obermeyer A. A. 1966. Potamogetonaceae. In: L. E. Codd, B. DE Winter \& H. B. Rycroft (eds.). Flora of South Africa 1, pp. 60-70. Cape and Transvaal Printers Ltd., Cape Town.

Ogden E. C. 1943. The Broad-leaved Species of Potamogeton. Rhodora 45: 57-105, 119-163, 171-214.

Olden J. D., Lawler J. J. \& Poff N. L. 2008. Machine Learning Methods without Tears: A Primer for Ecologists, Q. Rev. Biol. 83: 171-193.

Pilon J. \& Santamaria L. 2002. Clonal Variation in Morphological and Physiological Responses to Irradiance and Photoperiod for the Aquatic Angiosperm Potamogeton pectinatus. J Ecol. 90: 859-870.

PIP E. 1987. The Ecology of Potamogeton Species in Central North America. Hydrobiologia 153: 203-216.

Preston C. D. 1993. Irish Pondweeds IV. Potamogeton $\times$ lanceolatus Smith. Ir. Nat. J. 24(5): 213-218.

Preston C. D. 1995. Pondweeds of Great Britain and Ireland. BSBI Handbook no. 8, BSBI, London.

Preston C. D. \& Stewart N. F. 1994. Irish Pondweeds V. Potamogeton x suecicus K. Richter in Co Donegal. New to Ireland. Ir. Nat. J. 24(12): 485-489.

Reznicek A. A. \& Bobbette R. S. W. 1976. The taxonomy of Potamogeton subsection Hybridi in North America. Rhodora 78: 650-673.

Rooney N. \& KalfF J. 2000. Inter-annual variation in submerged macrophyte community biomass and distribution: The infl uence of temperature and lake morphometry. Aquat. Bot. 68: 321-335.

Santamaria L., Figuerola J., Pilon J., Mjelde M., Green A., De Boer T., King R. A. \& Gornall R. J. 2003. Plant Performance across Latitude: the Role of Plasticity and Local Adaptation in an Aquatic Plant. Ecology 84(9): 2454-2461.

Spence D. H. N. 1967. Factors Controlling the Distribution of Freshwater Macrophytes with Particular Reference to the Lochs of Scotland. J. Ecol. 55: 147-170.

SPENCE D. H. N. 1972. Light on freshwater macrophytes. Trans. Bot. Sot. Edinb. 41: 491-505.

Srivasta, D., Staicer, C. \& Freedman, B. 1995. Aquatic vegetation of Nova Scotia lakes differing in acidity and trophic status. Aquat. Bot. 51, 181-196.

Symoens J. J., Van de Velden J. \& Buescher P. 1979. Contribution a l'étude de la taxonomie et de la distribution de Potamogeton nodosus POIR. and Potamogeton thunbergii CHAM. \& SCHLECHTEND. en Afrique. Bull. Soc. Roy. Bot. Belgique 112: 79-95.

TER BraAk C. J. F. \& ŠMilauer P. 2002. CANOCO References Manual and User's Guide to Canoco for Windows: Software for Canonical Community Ordination (version 4.5). Microcomputer Power (Ithaca, NY, USA).
The Plant List 2013. The Plant List ver. 1.1. Accessed on: 15 May 2019. Available from: http://www.theplantlist. org/

Toivonen H., Huttunen P. 1995. Aquatic macrophytes and ecological gradients in 57 small lakes in southern Finland. Aquat. Bot. 51: 197-221.

Tur N. M. 1982. Revision del genero Potamogeton L. en la Argentina. Darwiniana 24: 217-266.

Tzvelev N. N. 1987. Sem. 136. Rdestovye - Potamogetonaceae Dumort. In: S. S. Kharkevich $[=$ S. S. CharKEVICZ] (ed.). Sosudistye rasteniya sovetskogo Dal'nego Vostoka [Plantea vasculares Orientis extreme Sovietici] 2, pp. 317-335. Nauka, Leningrad, (in Russian).

van den Berg A. E., Koole S. L. \& van der Wulp N. Y. 2003. Environmental preference and restoration: (How) are they related? Journal of Environmental Psychology 23:135-146.

Volobaev P. 1993. Nota de specie Potamogeton henningii A. Bann. (Potamogetonaceae) in Sibiria). Novosti Sist. Vyssh. Rast. 29: 5-8, (in Russian).

WANG Q. D., Zhang T. \& WANG J. B. 2007. Phylogenetic Relationships and Hybrid Origin of Potamogeton Species (Potamogetonaceae) Distributed in China: Insights from the Nuclear Ribosomal Internal Transcribed Spacer Sequence (ITS). P1. Syst. Evol. 267: 65-78.

Wiegleb G. 1983. A phytosociological study of the macrophytic vegetation of running waters in Western Lower Saxony (Federal Republic of Germany). Aquat. Bot. 17: 251-274.

Wiegleb G. 1984. A Study of the Habitat Conditions of the Macrophytic Vegetation in Selected River System in Western Lower Saxony (Federal Republic of Germany). Aquat. Bot. 18: 313-352.

Wiegleb G. 1990. The importance of Stem Anatomical Characters for the Systematics of the Genus Potamogeton L. Flora 184: 197-2018.

Wiegleb G. \& Kaplan Z. 1998. An account of the species of Potamogeton L. (Potamogetonaceae). Folia Geobot. 33: 241-316.

Yuzepchuk S. V. [= Juzepczuk S. V.] 1934. Sem. XVII. Rdestovyje - Potamogetonaceae ENGL. In: V. L. Komarov (ed.). Flora SSSR, 1, pp. 224-265. Leningrad, (in Russian).

ZalewsKa-GaŁosz J. 2002. Occurrence and distribution of Potamogeton hybrids (Potamogetonaceae) in Poland. Feddes Repert 113: 380-393.

ZalewsKa-Gatosz J. 2008. Rodzaj Potamogaton L. w Polsce - Taksonomia i rozmieszczenie. Wyd. Instytutu Botaniki Uniwersytetu Jagiellońskiego, Kraków, pp. 218, (in Polish).

Zalewska-Galosz J., Nowak A. \& Dajdok Z. 2012. Ecological Variation between Marginal and Central Populations of Potamogeton polygonifolius, a Rare and Endangered Species in Central Europe. J. Nat. Conserv. 20: 76-84.

ZANG T., WANG Q., Li W., Cheng Y. \& WANG J. 2008. Analisis of phylogenetic relationships of Potamogeton species in China based on chloroplast trnT-trnF sequences. Aquat. Bot. 89: 34-42. 